\title{
REGULARITY OF BMO WEAK SOLUTIONS TO NONLINEAR PARABOLIC SYSTEMS VIA HOMOTOPY
}

\author{
DUNG LE
}

\begin{abstract}
This paper introduces a new technique, using the so-called nonlinear heat approximation and BMO preserving homotopy, to investigate regularity properties of BMO weak solutions of strongly coupled nonlinear parabolic systems consisting of more than one equation defined on a domain of any dimension.
\end{abstract}

\section{INTRODUCTION}

We consider the following parabolic systems:

$$
u_{t}=\operatorname{div}(A(x, t, u, D u))+F(x, t, u, D u),
$$

in a domain $Q=\Omega \times(0, T) \subset \mathbb{R}^{n+1}$, with $\Omega$ being an open subset of $\mathbb{R}^{n}, n \geq 1$. The vector-valued unknown $u$ take values in $\mathbb{R}^{m}, m \geq 1$. Here, $u_{t}, D u$ denote respectively the temporal and the spatial derivatives of $u$. Here, $A: Q \times \mathbb{R}^{m} \times \mathbb{R}^{m n} \rightarrow \mathbb{R}^{m} \times \mathbb{R}^{n}$ and $F: Q \times \mathbb{R}^{m} \times \mathbb{R}^{m n} \rightarrow \mathbb{R}^{m}$ is a vector-valued function.

A weak solution $u$ to (1.1) is a function $u \in W_{2}^{1,0}\left(Q, \mathbb{R}^{m}\right)$ such that

$$
\iint_{Q}\left[-u \phi_{t}+A(x, t, u, D u) D \phi\right] d z=\iint_{Q} F(x, t, u, D u) \phi d z
$$

for all $\phi \in C_{c}^{1}\left(Q, \mathbb{R}^{m}\right)$. Here, we write $d z=d x d t$ and $W_{2}^{1,0}\left(Q, \mathbb{R}^{m}\right)$ is the set of vector-valued functions whose spatial weak derivatives are in $L^{2}(Q)$.

It is now a classical result (see [10, 16]) for the scalar case $(m=1)$ that bounded weak solutions to (1.1) are Hölder continuous and if $A, F$ are sufficiently smooth, then bounded weak solutions are classical. On the other hand, in the case of systems of equations (i.e. $m>1$ ), one cannot generally expect bounded weak solutions of (1.1) to be Hölder continuous everywhere (see counterexamples, e.g., in [5]). Partial regularity theory for (1.1), when $A$ is linear in $D u$, was considered in [2, 3], where it was shown that bounded weak solutions are Hölder continuous on a full measure subset of $Q$ and the Hausdörff dimension of their singular sets could be estimated. In a recent work, see [11, 14, we introduced the so-called linear $A$-heat approximation technique to investigate partial and everywhere regularity for degenerate parabolic systems. However, as pointed out in [1], it is crucial to establish everywhere Hölder continuity for bounded weak solutions for systems and to control their Hölder norms in order to discuss further important questions in applications (e.g.; see [18]) such as global existence and dynamics of the solutions. Works in these directions were reported in [6, 7, 8, 12, 17, 20, for systems satisfying

Received by the editors June 10, 2011 and, in revised form, September 26, 2011.

2010 Mathematics Subject Classification. Primary 35K65, 35B65.

Key words and phrases. Parabolic systems, Hölder regularity, BMO weak solutions.

The author was partially supported by NSF grant DMS0707229. 
intrinsic structural conditions on $A, F$ of (1.1) and seem very hard to verify unless (1.1) consists of only two equations (i.e., $m=2$; see [12]) or the dimension $n$ is small (e.g., $n \leq 4$ in [17] or $n=2$ in [6]). The method and conditions in [8] cannot apply here due to the presence of $u$ in $A$ and $u$ is only assumed to have bounded BMO norm.

In this work, we seek for everywhere regularity for systems such as (1.1) given on a domain of $\mathbb{R}^{n}$ and consisting of $m$ equations with $m, n$ being arbitrary while structural conditions on $A, F$ are much less restrictive. For the sake of simplicity, we will assume in this paper that $A, F$ depend only on $u, D u$. However, the case when they depend smoothly on $x, t$ can also be treated modulo minor modifications.

The main theme of our approach here is to embed the considered system (1.1) into a family of nonlinear systems such that if it is continuously homotopic to a member of the family whose everywhere regularity is known, then the same regularity can be carried over to (1.1). In this homotopy, the main assumption is that a certain $\mathrm{BMO}$, instead of $L^{\infty}$, maximum principle remains. As we deal mainly with weak solutions, such a BMO principle is obviously more reasonable, and perhaps easier to obtain under much weaker hypotheses, than its $L^{\infty}$ versions (see [9]). In fact, we will need only such a BMO maximum principle for regular solutions to the Cauchy problem of (1.1) with boundary and initial data being smooth. Works in this direction are in progress and some results on BMO maximum principles for systems such as (1.1) will be reported in a forthcoming paper. Meanwhile, some $L^{\infty}$ maximum principles were studied in an earlier work [13] on nearly triangular systems.

Thus, we will consider a family of parabolic systems parameterized by $\nu \in[0,1]$ :

$$
u_{t}=\operatorname{div}(A(\nu, u, D u))+f(\nu, u, D u) \text {, in } Q=\Omega \times(0, T) \subset \mathbb{R}^{n+1} .
$$

Assume that (1.1) becomes the above system when $\nu=1$ and that Hölder continuity results are known for the system when $\nu=0$. It is well known that Hölder continuity of a vector-valued function $u$ is equivalent to a certain decay estimate for its mean oscillation, property D) described in Section 2. We then consider a subset $I$ of parameters in $[0,1]$ where BMO weak solutions of the above system satisfy this property. The main goal is then to prove that $I$ is both open and closed in $[0,1]$ so that $I=[0,1]$ and the desired Hölder continuity for solutions to (1.1) is obtained. Our first two main results concerning the open and closed properties of $I$ will be presented under two sets of conditions as they will be established by using different tools, and they may be independently of interest in applications.

The main vehicles in the proof of $I$ that are open are Proposition 4.1 and Proposition 4.4, which are nonlinear versions of the (linear) heat approximation method in [11. Basically, they assert that if a vector-valued function $u$ almost and weakly solves a system such as (1.2), with $\nu \in I$, then it can be approximated in a certain controllable way by a solution $v$ of the system. By this, regularity properties of $v$ can be carried over to $u$. The proof of these nonlinear approximation versions is not a simple extension of that for the constant coefficient linear case in [1] and much more is involved. One of the main obstacles here is that the existence of the approximating solution $v$ for the Cauchy problem involving a nonlinear system such as (1.2) is not available if the data $u$ is not smooth, but only BMO. We will have to mollify $u$ to get $v$ and several technicalities will appear.

On the other hand, the above argument is local by nature and cannot be used to prove that $I$ is closed as it lacks certain uniform estimates in order to show that 
limits of a sequence of regular solutions are also regular. To this end, we will use a different approach deriving uniform and global estimates for the integrals of spatial derivatives of regular solutions with uniform bounded BMO norms.

Combining the above two results we obtain regularity properties for BMO weak solutions to (1.1) via the described homotopy. Obviously, we need to determine candidates for regular systems to which a given (1.1) can be homotopic. Apparently, a natural candidate of such systems is

$$
u_{t}=\operatorname{div}(A(0, D u))+F(0, D u), \quad \nu \in[0,1],
$$

to which (1.1) can be continuously transformed via

$$
u_{t}=\operatorname{div}(A(\nu u, D u))+F(\nu u, D u), \quad \nu \in[0,1] .
$$

We will study regularity results of (1.3) when $A$ is independent of $u$. The results in this direction recover those of Necas and Sverak [17] when $n \leq 4$ and further extend to the case for larger $n$.

The paper is organized as follows. In Section 2, we will introduce notation and discuss in detail our hypotheses and main theorems. Section 3 collects technical lemmas. Section 4 presents our main vehicles: the nonlinear approximation results. The proof that $I$ is open will be given in Section 5. Finally, Section 6 details the proof of $I$ being closed as well as the study of (1.3) and concludes our paper.

\section{PReliminaries AND MAIN TheOREMS}

Throughout this paper, for some $z_{0}=\left(x_{0}, t_{0}\right) \in \mathbb{R}^{n+1}, Q_{R}\left(z_{0}\right)$ denotes the parabolic cylinder centered at $z_{0}$ with radius $R$. That is, $Q_{R}\left(z_{0}\right)=B_{R}\left(x_{0}\right) \times\left[t_{0}-\right.$ $\left.R^{2}, t_{0}\right]$. We also abbreviate by $S_{R}\left(z_{0}\right)=B_{R}\left(x_{0}\right) \times\left\{t_{0}-R^{2}\right\} \cup \partial B_{R}\left(x_{0}\right) \times\left[t_{0}-R^{2}, t_{0}\right]$, the parabolic boundary of $Q_{R}\left(z_{0}\right)$. If the center $z_{0}$ was understood, we would simply write $Q_{R}, S_{R}$ for $Q_{R}\left(z_{0}\right)$ and $S_{R}\left(z_{0}\right)$ respectively.

For any integrable function $u: Q_{R} \rightarrow \mathbb{R}^{m}$ and any measurable subset $A$ of $Q_{R}$, we write

$$
u_{A}=\frac{1}{|A|} \int_{A} u(z) d z=\oiint_{A} u(z) d z .
$$

If $A$ is a cylinder $Q_{R}=Q_{R}\left(z_{0}\right)$ and there is no ambiguity, we simply write $u_{R}=$ $u_{Q_{R}}$.

We consider the space $V\left(Q_{R}\right)=V\left(Q_{R}, \mathbb{R}^{m}\right)$ (see [10] ) of vector-valued functions $u: Q_{R} \rightarrow \mathbb{R}^{m}$ with finite (normalized) norm $\|\cdot\|_{V\left(Q_{R}\right)}$ defined by

$$
\|u\|_{V\left(Q_{R}\right)}^{2}=\sup _{t \in\left[-R^{2}+t_{0}, t_{0}\right]} R^{-n} \int_{B_{R}}|u(x, t)|^{2} d x+R^{2} \oiiint_{Q_{R}}|D u|^{2} d z .
$$

By $V_{0}\left(Q_{R}\right)$ we denote the closure of $C_{0}^{1}\left(Q_{R}\right)$ in $V\left(Q_{R}\right)$ with respect to the above norm.

For any cylinder $Q$ in $\mathbb{R}^{n+1}$, the local BMO space $B M O(Q)=B M O\left(Q, \mathbb{R}^{m}\right)$ consists of vector-valued functions $u$ such that

$$
\|u\|_{B M O(Q)}^{2}=\sup _{Q_{R}} f \int_{Q_{R} \cap Q}\left|u-u_{Q_{R} \cap Q}\right|^{2} d z<\infty .
$$

We also make use of the Morrey and Campanato spaces $L^{p, \lambda}$ and $\mathcal{L}^{p, \lambda}$ :

$$
L^{p, \lambda}(Q)=\left\{u: u \in L^{p}\left(Q, \mathbb{R}^{m}\right) \text { and }\|u\|_{p, \lambda}^{p}=\sup _{\substack{z \in Q \\ \rho>0}} \rho^{-\lambda} \iint_{Q_{\rho}(z) \cap Q}|u|^{p} d z<\infty\right\}
$$


and

$$
\mathcal{L}^{p, \lambda}(Q)=\left\{u: u \in L^{p}\left(Q, \mathbb{R}^{m}\right) \text { and }[u]_{p, \lambda}^{p}=\sup _{\substack{z \in Q \\ \rho>0}} \rho^{-\lambda} \iint_{Q_{\rho}(z) \cap Q}\left|u-u_{z, \rho}\right|^{p} d z<\infty\right\},
$$

where $u_{z, \rho}=\int_{Q_{\rho}(z) \cap Q} u d z$.

As our results are local in nature, without loss of generality, we will simply consider $Q$ as the unit parabolic cylinder $B_{1}(0) \times[-1,0]$ throughout this paper. We then consider a family of systems

$$
u_{t}=\operatorname{div}(A(\nu, u, D u))+F(\nu, u, D u) \text {, in } Q=B_{1} \times(-1,0) \subset \mathbb{R}^{n+1} \text { and } \nu \in[0,1] \text {. }
$$

By a BMO weak solution $u$ to this system we mean a BMO function $u$ satisfying $\iint_{Q}\left[-u \phi_{t}+\langle A(\nu, u, D u), D \phi\rangle\right] d z-\iint_{Q} F(\nu, u, D u) \phi d z=0, \quad \forall \phi \in C_{0}^{1}\left(Q, \mathbb{R}^{m}\right)$.

In order to investigate the Hölder regularity of weak solutions, we recall a result in 3 showing that a vector-valued function $u$ is Hölder continuous if and only if it satisfies a mean oscillation decay estimate described in the following property.

D) For some $\tau_{0}, \alpha \in(0,1)$ and any $Q_{R}=Q_{R}(x, t) \subset Q$, there is a constant $C\left(\tau_{0}\right)$ such that

$$
\oiiint_{Q_{\tau R}}\left|u-u_{\tau R}\right|^{2} d z \leq C\left(\tau_{0}\right) \tau^{\alpha} \int_{Q_{R}}\left|u-u_{R}\right|^{2} d z, \quad \forall \tau \in\left(0, \tau_{0}\right),
$$

where $u_{R}=\oiiint_{Q_{R}} u d z$.

Our first main result shows that if (2.2), for some $\nu$, is a "nice" system in the sense that its BMO weak solutions are Hölder continuous, then "near by" systems are also nice. The main assumption here is that the "nice" system satisfy a certain BMO maximum principle.

To be more precise, let us describe our assumptions in detail here. We first suppose that the family of systems (2.2) contains at least a "nice" one so that the set of parameters $\nu$, with which the system is nice, makes up a nonempty set $I$.

I) There is a nonempty set $I \subset[0,1]$ such that for any $\nu$ in $I$ the decay property D) holds for any BMO weak solution to (2.2). That is, if $u$ is a weak solution of (2.2),$\nu \in I$, with $\|u\|_{B M O(Q)} \leq M$, then there exists $\alpha(M)>0$ such that for any $\alpha \in(0, \alpha(M))$ there is $\tau(M, \alpha)>0$ and the solution $u$ satisfies the decay estimate (2.3) for some constant $C(M, \alpha)$ and $\tau \in(0, \tau(M, \alpha))$.

We then consider the following structural assumptions on the matrices $A(\nu, u, D u)$ and the vector-valued functions $f(\nu, u, D u)$.

H.1) (Uniform ellipticity) There are positive constants $\lambda, \Lambda$ such that for all $\nu \in[0,1], u \in \mathbb{R}^{m}$ and $\zeta, \eta \in \mathbb{R}^{n m}$,

$$
\langle A(\nu, u, \zeta), \zeta\rangle \geq \lambda|\zeta|^{2}, \quad|\langle A(\nu, u, \zeta), \eta\rangle| \leq \Lambda|\zeta||\eta| .
$$


Furthermore, $\frac{\partial A}{\partial \zeta}(\nu, u, \zeta)$ exists and

$$
\begin{gathered}
\left\langle\frac{\partial A}{\partial \zeta}(\nu, u, \zeta) \eta, \eta\right\rangle \geq \lambda|\eta|^{2}, \\
|A(\nu, u, \zeta)-A(\nu, v, \zeta)| \leq C|u-v||\zeta|
\end{gathered}
$$

for some positive constant $C$.

H.2) $F:[0,1] \times \mathbb{R}^{m} \times \mathbb{R}^{n m} \rightarrow \mathbb{R}^{m}$ and $|f(\nu, u, D u)| \leq C(f+|D u|)$ for some positive constant $C$ and a function $f \in L^{r, n+2-r}(Q)$ with $r>2$. Moreover,

$$
|F(\nu, u, \xi)-F(\nu, v, \eta)| \leq C|u-v|+C|\xi-\eta| .
$$

H.3) $A(\nu, u, \zeta), F(\nu, u, \zeta)$ are Hölder in $\nu \in[0,1]$ and

$$
\begin{aligned}
|A(\nu, u, \zeta)-A(\mu, u, \zeta)| & \leq C|\nu-\mu|^{\theta}|\zeta| \\
|F(\nu, u, \zeta)-F(\mu, u, \zeta)| & \leq C|\nu-\mu|^{\theta}(|f|+1)|\zeta|
\end{aligned}
$$

for some $C, \theta, l>0$ and any $u \in \mathbb{R}^{m}, \zeta \in \mathbb{R}^{n m}$. The function $f$ in the above is described in H.2).

H.4) (Existence) For $\nu \in I$, the set of "nice" parameters, and any cylinder $Q_{R}=Q_{R}(x, t) \subset Q$, and any vector-valued function $g \in C^{1}\left(Q_{R}\right)$, the system

$$
\left\{\begin{array}{l}
\iint_{Q_{R}}\left[-u \phi_{t}+\langle A(\nu, u, D u), D \phi\rangle-\langle F(\nu, u, D u), \phi\rangle\right] d z=0, \quad \forall \phi \in C_{0}^{1}\left(Q_{R}, \mathbb{R}^{m}\right), \\
u=g \text { on } S_{Q_{R}}
\end{array}\right.
$$

has a BMO weak solution $u$ (see also Remark 2.1 below).

H.5) (Uniform BMO estimate) For any $\nu \in I$, if $g \in C^{1}\left(Q_{R}\right)$, then there is a constant $C\left(\|g\|_{B M O\left(Q_{R}\right)}\right)$ such that any weak solution $u$ to the system (2.8) is BMO and satisfies the estimate $\|u\|_{B M O\left(Q_{R}\right)} \leq C\left(\|g\|_{B M O\left(Q_{R}\right)}\right)$.

The assumption that the function $f$ belongs to the Morrey space described in H.2) comes naturally in conjunction with the BMO condition H.5). In fact, by testing the systems with $\left(u-u_{r}\right) \phi^{2}$, where $\phi$ is a cutoff function for any two concentric cylinders $Q_{r}$ and $Q_{2 r}$ of $Q$, one can quickly see that $f$ should be in such a Morrey space.

Remark 2.1. The condition H.4) differs in this work from those using linear heat approximation arguments, where (2.8) is a linear system with constant coefficients and H.4) is granted even when the Cauchy data $g$ is not smooth, which is not the case here since our reference systems are nonlinear and our Cauchy data are only BMO. However, the work [1] provided local existence and uniqueness results for the Cauchy problem (2.8) if the boundary data is $C^{1}$ smooth and the matrices $A(\nu, u, D u)$ are regular elliptic. Therefore the assumption H.4) on (2.8), when $\nu$ is in the "nice" set $I$, is justified if $Q_{R}$ is replaced by some cylinder $B_{R} \times\left[t_{0}-h, t_{0}\right]$ with $h$ being sufficiently small. However, it was also shown in [1] that if one can establish a priori estimates for the Hölder norms of these solutions in $Q_{R}$, then they also (uniquely) exist in $Q_{R}$. Hence, when $\nu \in I$, the condition H.4) is somewhat just a consequence of I), combining with H.5) and our arguments later on, which guarantees such required estimates for Hölder norms of the solutions via the decay property D). In order to use H.4) in the proof of our approximation 
results in Section 4, we have to mollify the approximated solution, and more delicate arguments will be needed in our proof.

Remark 2.2. From the proof we can easily see that the constants $\lambda, \Lambda$ could be allowed to depend on $u$. We need only assume that the diffusion matrix $A$ is regularly elliptic; i.e., the ratio $c_{1} \leq \lambda / \Lambda \leq c_{2}$ and $\lambda \geq c_{3}$ for some positive constants $c_{i}$.

Our first main result then asserts that the set $I$, where the property D) holds, is open.

Theorem 2.3. Suppose that I) and H.1)-H.5) hold. Then I is open in the usual topology of $[0,1]$.

In fact, as corollaries to the above theorem, if $A, F$ are smoother (e.g., $C^{1}$ in their variables), then BMO solutions to nice systems (with $\nu$ being in $I$ ) are $C_{l o c}^{1}(Q)$. We will discuss these types of results in Section 5 .

Next, we will give conditions for the set $I$ to be closed in $[0,1]$. To this end, we take a sequence $\left\{\nu_{k}\right\}$ in $I$ such that $\nu_{k} \rightarrow \mu$ and we will show that $\mu \in I$. We first require that any weak BMO solution $u$ to (2.2) can be weakly approximated by "nice" solutions.

II) For each $\nu \in I$, the system (2.2) satisfies the existence condition H.4) and BMO maximum principle H.5). Moreover, if $\nu_{k} \subset I$ and $\nu_{k} \rightarrow \mu$, then for any weak BMO solution $u$ to (2.2) with $\nu=\mu$ there is a sequence $\left\{v_{k}\right\}$ of $C^{1}$ solutions to (2.2), with $\nu=\nu_{k}$, such that $D v_{k}$ converges weakly to $D u$ in $L^{1}(Q)$ and the BMO norms of $v_{k}$ are bounded uniformly in terms of that of $u$.

Remark 2.4. Although it will be shown in Section 4 that the above assumption holds under H.1)-H.5) via nonlinear heat approximations, we state II) here for our next result so that it is independent of Theorem 2.3. Apparently, II) could also be verified by other means using weaker assumptions than H.1)-H.5).

We then consider $\nu \in I$ and a $C^{1}$ solution $v$ to

$$
\begin{cases}v_{t}=\operatorname{div}(A(\nu, v, D v))+F(\nu, v, D v) & \text { in } Q_{1} \\ v=g & \text { on } S_{1}\end{cases}
$$

The boundary condition $g$ is assumed to be smooth. By II), the above system satisfies the BMO maximum principle and $\|v\|_{B M O(Q)} \leq C\left(\|g\|_{B M O(Q)}\right)$. Since $v$ is bounded in $Q_{\frac{3}{4}}$, we can define

$$
M_{\nu, v}=\sup _{Q_{\frac{3}{4}}}|v| \text { and } M_{B}=\|v\|_{B M O\left(Q_{1}\right)} .
$$

Note that $M_{\nu, v}$ is not a uniform bound for every solution $v$ to (2.9). Meanwhile, by the assumed maximum principle for BMO norms in H.5), $M_{B}$ can be taken to be a fixed constant depending only on the BMO norm of $g$.

We then impose the following assumptions on the structure of (2.9).

M.1) For any $\nu \in[0,1]$, the matrix $\frac{\partial A}{\partial \zeta}(\nu, u, \zeta)$ is uniformly elliptic. Let $\lambda_{\nu, v}, \Lambda_{\nu, v}$ be the ellipticity constants for the matrix $\left(A_{k l}^{i j}\right)=\frac{\partial A}{\partial \xi}(\nu, v, \xi)$, 
that is,

$$
\sum_{i, j=1}^{m} \sum_{k, l=1}^{n} A_{k l}^{i j} \eta_{k}^{i} \eta_{l}^{j} \geq \lambda_{\nu, v}|\eta|^{2}, \quad \sum_{i, k}\left(\sum_{j, l} A_{k l}^{i j} \eta_{l}^{j}\right)^{2} \leq \Lambda_{\nu, v}^{2}|\eta|^{2} .
$$

If $n>2$, we also assume that

$$
\sup \left\{\frac{\Lambda_{\nu, v}}{\lambda_{\nu, v}}: \quad v \text { is a bounded function }\right\}<\frac{n}{n-2} .
$$

M.2) For every $\nu$ in $I$ and any BMO weak solution $v$ to (2.9), there exists a positive constant $a_{\nu, v}$ such that

$$
\left|\frac{\partial A}{\partial v}(\nu, v, \xi)\right| \leq a_{\nu, v}|\xi| \text { with } 2 a_{\nu, v} M_{\nu, v}(n+1)<\sigma_{0} \widehat{\lambda}_{\nu, v}
$$

where $\sigma_{0}$ is a fixed number in $(0,1)$ and

$$
\widehat{\lambda}_{\nu, v}=\left(1-\delta^{2}\right) \lambda_{\nu, v} \text { and } \delta=\frac{n-2}{n} \sup \left\{\frac{\Lambda_{\nu, v}}{\lambda_{\nu, v}}\right\} .
$$

M.3) $F(\nu, v, \xi)$ is a $C^{1}$ function in $v, \xi$ and for some positive constants $C, l, \varepsilon_{0}$, we have

$$
\left|\frac{\partial F}{\partial v}(\nu, v, \xi)\right| \leq C\left(1+|v|^{l}+|\xi|\right), \quad\left|\frac{\partial F}{\partial \xi}(\nu, v, \xi)\right| \leq C\left(1+C|v|^{l}\right) .
$$

Note that $\widehat{\lambda}_{\nu, v}>0$ due to (2.11). Meanwhile, (2.11) requires that the principal eigenvalues $\Lambda_{\nu, v}, \lambda_{\nu, v}$ of $\frac{\partial A}{\partial \xi}$ are not too far apart (when $n>2$ ). On the other hand, situations for the second condition in (2.12) to hold need further discussion. Among them could be the case when $A=C \xi+a_{1}(v) \xi+a_{2}(v, \xi)$, where $C$ is a constant matrix. If $a_{2}$ is negligible, then $\frac{\partial A}{\partial v} \sim \frac{\partial a_{1}}{\partial v} \xi$ and the term $a_{\nu, v} M_{\nu, v} \sim\left|a_{1}(v)\right|$. (2.12) is then possible if the constant coefficient $C$ dominates $a_{1}(v)$ and mainly determines the ellipticity constant $\lambda_{\nu, v}$.

We remark here that our conditions M.1) and M.2) concern the relation between the principal eigenvalues of $\frac{\partial A}{\partial \xi}$ and they should be compared with those used in [8]. First of all, the method in [8] cannot apply to our more general setting due to the presence of $u$, which is not assumed to be bounded, in $A$. Here, we assume only that the matrix $A$ is regularly elliptic, i.e. $\Lambda_{\nu, v}, \lambda_{\nu, v}$ can depend on $u$ and be unbounded (thus [8, (15)] cannot be verified here) and that the quotient $\Lambda_{\nu, v} / \lambda_{\nu, v}$ is bounded from above and below (recall Remark 2.2). Secondly, our assumptions make use of the ratio $\Lambda_{\nu, v} / \lambda_{\nu, v}$ rather than the constant $K$ describing the dispersion of the eigenvalues as in [8, (*) and (12)].

Our next main result then reads

Theorem 2.5. Assume the conditions II) and M.1)-M.3). The set I is closed in $[0,1]$.

Combining with the results of the previous theorem, and Remark 2.4, we then have

Theorem 2.6. Assume I), H.1)-H.5) and M.1)-M.3). Then $I=[0,1]$. Thus, BMO weak solutions are Hölder continuous. 
Finally, we would like to provide examples of "nice" (or reference) systems such that $I$ is not empty and the homotopy (2.2) could be realized. A natural candidate of such a family can be

$$
u_{t}=\operatorname{div}(A(\nu u, D u))+F(\nu u, D u), \quad \nu \in[0,1] .
$$

This leads us to the study of the following reference system when $\nu=0$ :

$$
u_{t}=\operatorname{div}(A(D u))+F(u, D u) .
$$

The proof of Theorem 2.5 can be adapted here, without using the condition M.2), to prove the following result.

Theorem 2.7. If either $n>4$ and M.1) holds or $n \leq 4$ and (2.10) holds, then BMO weak solutions to (2.14) are Hölder continuous.

We should remark that the above theorem does not require the dispersion condition (2.11) on the eigenvalues of $\frac{\partial A}{\partial \xi}$ but its ellipticity when $n \leq 4$.

\section{Some technical LEMmas}

We collect some technical lemmas in this section. We first recall the following Sobolev imbedding inequality (see [10]):

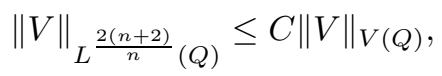

where $C$ is a constant depending only on $n$.

As we will deal with BMO functions, the following fact is well known (4, Corollary 2.2, p. 57]) and states that they are almost bounded.

Lemma 3.1. If $u \in B M O(Q)$, then $u \in L^{p}(Q)$ for any $p \geq 1$ and it follows for any $Q_{R} \subset Q$ that

$$
\oiiint_{Q_{R}}\left|u-u_{Q}\right|^{p} d z \leq C_{p}\|u\|_{B M O(Q)}^{p} .
$$

The next lemma is a simple calculation fact.

Lemma 3.2. Let $Q$ be $Q_{1}(0)=B \times[-1,0]$ and $u: Q \rightarrow \mathbb{R}^{m}$ be in $V(Q)$. Assume that there is a function $G \in L^{1}(Q)$ such that

$$
\left|\iint_{Q} u \phi_{t} d z\right| \leq C \iint_{B \times(s, r)}|G||D \phi| d z \quad \forall \phi \in C_{0}^{1}(Q) .
$$

Then, for any $l>(n+2) / 2$ and $-1<s<r<0$, there exists a constant $C$ such that

$$
\|u(\cdot, r)-u(\cdot, s)\|_{W^{-l, 2}(B)} \leq C \int_{s}^{r} \int_{B}|G| d x d t .
$$

Proof. For $\varepsilon>0$, take $\phi$ in (3.2) to be $\psi(x) \eta(t)$, where $\psi \in C_{0}^{1}(B)$ and $\eta \equiv 1$ in $(s, r), \eta$ is linear in $(s-\varepsilon, s)$ and $(r, r+\varepsilon)$, and $\eta$ is zero elsewhere. We see that

$$
\left|\int_{B}\left(\frac{1}{\varepsilon} \int_{s-\varepsilon}^{s} u d t-\frac{1}{\varepsilon} \int_{r}^{r+\varepsilon} u d t\right) \psi d x\right| \leq C\left(\iint_{B \times(s-\varepsilon, r+\varepsilon)}|G \eta| d z\right)\|\psi\|_{C_{0}^{1}(B)} .
$$


For any $l>(n+2) / 2$, we have $W_{0}^{l, 2}(B) \subset C_{0}^{1}(B)$ and $\|\psi\|_{C_{0}^{1}(B)} \leq C\|\psi\|_{W^{l, 2}(B)}$. Therefore, by letting $\varepsilon \rightarrow 0$ in the above, we obtain the following estimate:

$\left|\int_{B}(u(x, s)-u(x, r)) \psi(x) d x\right| \leq C\left(\iint_{B \times(s, r)}|G| d z\right)\|\psi\|_{W_{0}^{l, 2}(B)} \quad \forall \psi \in W_{0}^{l, 2}(B)$.

This gives (3.3).

We then have the following compactness result for the space $V(Q)$.

Lemma 3.3. Let $Q$ be $Q_{1}(0)=B \times[-1,0]$ with $B=B_{1}$. Consider two sequences of functions $\left\{v_{k}\right\} \subset V(Q)$ and $\left\{G_{k}\right\} \subset L^{2}(Q)$. Assume that there are a constant $M$ and some sequence of positive reals $\varepsilon_{k}$ converging to 0 such that:

c.1) For all $k$,

$$
\iint_{Q}\left(v_{k}^{2}+\left|D v_{k}\right|^{2}\right) d z, \quad\left\|G_{k}\right\|_{L^{2}(Q)} \leq M
$$

c.2) For any $\phi \in C_{0}^{1}(Q)$ we have

$$
\left|\iint_{Q} v_{k} \phi_{t} d z\right| \leq C \iint_{Q}\left|G_{k}\right||D \phi| d z+\varepsilon_{k} \sup _{t}\|\phi(\cdot, t)\|_{C^{1}(B)} .
$$

Then $\left\{v_{k}\right\}$ has a subsequence that converges in $L^{2}(Q)$.

Proof. Arguing as in Lemma 3.2 with $G$ being replaced by $G_{k}+\varepsilon_{k}$, we obtain

$$
\left\|v_{k}(\cdot, r)-v_{k}(\cdot, s)\right\|_{W^{-l, 2}(B)} \leq C\left[\int_{s}^{r} \int_{B}\left|G_{k}\right| d x d t+\varepsilon_{k}\right] .
$$

Using the Hölder inequality, we see that

$$
\left\|v_{k}(\cdot, s)-v_{k}(\cdot, r)\right\|_{W^{-l, 2}(B)} \leq C\left(\sqrt[2]{r-s}+\varepsilon_{k}\right) .
$$

Interpolating $L^{2}\left(B, \mathbb{R}^{N}\right)$ between $W^{1,2}\left(B, \mathbb{R}^{N}\right)$ and $W^{-l, 2}\left(B, \mathbb{R}^{N}\right)$ it follows for $\mu>0$ that

$$
\begin{aligned}
& \int_{-1}^{-h}\left\|v_{k}(\cdot, t+h)-v_{k}(\cdot, t)\right\|_{L^{2}(B)}^{2} d t \\
& \leq \quad \mu \int_{-1}^{-h}\left\|v_{k}(\cdot, t+h)-v_{k}(\cdot, t)\right\|_{W^{1,2}(B)}^{2} d t \\
& \quad+C(\mu) \int_{-1}^{-h}\left\|v_{k}(\cdot, t+h)-v_{k}(\cdot, t)\right\|_{W^{-l, 2}(B)}^{2} d t \\
& \quad \leq \quad 2 \mu \int_{-1}^{0}\left\|v_{k}(\cdot, t)\right\|_{W^{1,2}(B)}^{2} d t+C(\mu) C\left(h+\varepsilon_{k}\right) \mid \leq 2 \mu+C(\mu) C\left(h+\varepsilon_{k}\right) .
\end{aligned}
$$

Thus, for any given $\varepsilon>0$, by choosing $\mu$ small and then $h$ small, $k$ large, we see that

$$
\int_{-1}^{-h}\left\|v_{k}(\cdot, t+h)-v_{k}(\cdot, t)\right\|_{L^{2}(B)}^{2} d t \leq \varepsilon
$$

In addition, for any $t_{1}, t_{2}$ in $(-1,0)$, we see by c.1) that

$$
\left\|\int_{t_{1}}^{t_{2}} v_{k}(\cdot, s) d s\right\|_{W^{1,2}(B)}^{2} \leq \iint_{Q}\left(v_{k}^{2}+\left|D v_{k}\right|^{2}\right) d z \leq M
$$


Thus, the functions $\int_{t_{1}}^{t_{2}} v_{k}(\cdot, s) d s$ belong to a fixed compact set $K$ in $L^{2}(B)$. Let $F=\left\{v_{k}\right\}$. We just established the following:

$$
\begin{gathered}
\forall t_{1}, t_{2} \in(-1,0) \text { and } t_{1}<t_{2}, \quad\left\{\int_{t_{1}}^{t_{2}} f(t) d t: f \in F\right\} \subset K, \\
\int_{-1}^{-h}\|f(t+h)-f(t)\|_{L^{2}(B)}^{2} d t \leq O(h), \quad \forall f \in F .
\end{gathered}
$$

According to a compactness result by Simon [19], we see that $F$ is precompact in $L^{2}\left([-1,0], L^{2}(B)\right)$.

Another consequence of Lemma 3.2 is the following reverse Hölder inequality.

Lemma 3.4. Consider a cylinder $Q_{R}=B_{R} \times\left(-R^{2}, 0\right)$ and a function $u$ in $V\left(Q_{R}\right)$. Assume that there are functions $G, F \in L^{1}\left(Q_{R}\right)$ such that

$$
\left|\iint_{Q_{R}} u \phi_{t} d z\right| \leq C \iint_{Q_{R}}(|G||D \phi|+|F| \phi) d z \quad \forall \phi \in C_{0}^{1}\left(Q_{R}\right) .
$$

$$
\begin{aligned}
\frac{1}{R^{2}} \oiint_{Q_{R}}\left|u-u(\cdot, t)_{R}\right|^{2} d z \leq & \varepsilon \oiint_{Q_{R}}|D u|^{2} d z+(C+\varepsilon)\left(\oiint_{Q_{R}}|D u|^{q} d z\right)^{\frac{2}{q}} \\
& +C(\varepsilon)\left(\oiiint_{Q_{R}}(|G|+R|F|) d z\right)^{2} .
\end{aligned}
$$

Proof. Let $s, t$ be in $\left(-R^{2}, 0\right)$. By scaling and arguing as in the proof of Lemma 3.2 . we easily see that

$$
\left|\int_{B_{R}}(u(\cdot, s)-u(\cdot, t)) \psi(x) d x\right| \leq C R^{\frac{2 l-2-n}{2}}\left(\iint_{Q_{R}}(|G|+R|F|) d z\right)\|\psi\|_{W_{0}^{l, 2}\left(B_{R}\right)}
$$

for all $\psi \in W_{0}^{l, 2}\left(B_{R}\right)$. Set $H(t, s, \cdot)=u(\cdot, s)-u(\cdot, t)$. The above reads

$$
\|H(t, s, \cdot)\|_{W^{-l, 2}\left(B_{R}\right)} \leq C R^{\frac{2 l-2-n}{2}}\left(\iint_{Q_{R}}(|G|+R|F|) d z\right) .
$$

Let $q$ be such that $2_{*}=2 n /(n+2) \leq q<2$. We have $W^{1, q}\left(B_{R}\right) \subset L^{2}\left(B_{R}\right) \subset$ $W^{-l, 2}\left(B_{R}\right)$. Because $W^{1, q}\left(B_{R}\right)$ is compactly imbedded in $L^{2}\left(B_{R}\right)$, a simple argument by contradiction gives the following interpolation inequality:

$R^{-n}\|\phi\|_{L^{2}\left(B_{R}\right)}^{2} \leq \varepsilon R^{\left(1-\frac{n}{q}\right) 2}\|D \phi\|_{L^{q}\left(B_{R}\right)}^{2}+C(\varepsilon) R^{-2 l-n}\|\phi\|_{W^{-l, 2}\left(B_{R}\right)}^{2}, \forall \phi \in W^{1, q}\left(B_{R}\right)$.

Applying this to $H(t, s, \cdot)$ and using (3.6), we have

$$
\begin{aligned}
R^{-n}\|H(t, s, \cdot)\|_{L^{2}\left(B_{R}\right)}^{2} \leq & \varepsilon R^{\left(1-\frac{n}{q}\right) 2}\|D H(t, s, \cdot)\|_{L^{q}\left(B_{R}\right)}^{2} \\
& +C(\varepsilon) R^{-2-2 n}\left(\iint_{Q_{R}}(|G|+R|F|) d z\right)^{2} .
\end{aligned}
$$

It is easy to see that we can choose $s$ such that

$$
\int_{B_{R}}|D u(x, s)|^{q} d x \leq \frac{1}{R^{2}} \iint_{Q_{R}}|D u(x, t)|^{q} d z .
$$


We then fix such an $s$ from now on and note that

$$
\|D u(\cdot, s)\|_{L^{q}\left(B_{R}\right)}^{2} \leq\left(\frac{1}{R^{2}} \iint_{Q_{R}}|D u(x, t)|^{q} d z\right)^{\frac{2}{q}}=R^{-4 / q}\left(\iint_{Q_{R}}|D u|^{q} d z\right)^{\frac{2}{q}} .
$$

On the other hand, by Sobolev-Poincaré's inequality, we also have

$$
\begin{aligned}
R^{-n}\left\|u(\cdot, s)-u(\cdot, s)_{R}\right\|_{L^{2}\left(B_{R}\right)}^{2} & \leq C R^{\left(1-\frac{n}{q}\right) 2}\|D u(\cdot, s)\|_{L^{q}\left(B_{R}\right)}^{2} \\
& \leq C R^{2}\left(\oiint_{Q_{R}}|D u|^{q} d z\right)^{\frac{2}{q}} .
\end{aligned}
$$

From the facts that

$$
\left\|u(\cdot, t)-u(\cdot, t)_{R}\right\|_{L^{2}\left(B_{R}\right)}^{2} \leq\|u(\cdot, t)-u(\cdot, s)\|_{L^{2}\left(B_{R}\right)}^{2}+\left\|u(\cdot, s)-u(\cdot, s)_{R}\right\|_{L^{2}\left(B_{R}\right)}^{2}
$$

and that $|D H(t, s, \cdot)| \leq|D u(\cdot, t)|+|D u(\cdot, s)|$, we derive from the above and (3.7) that

$$
\begin{aligned}
\frac{1}{R^{n}}\left\|u(\cdot, t)-u(\cdot, t)_{R}\right\|_{L^{2}\left(B_{R}\right)}^{2} \leq & \varepsilon R^{2-n}\|D u(\cdot, t)\|_{L^{2}\left(B_{R}\right)}^{2} \\
& +(C+\varepsilon) C R^{2}\left(\int_{Q_{R}}|D u|^{q} d z\right)^{\frac{2}{q}} \\
& +C(\varepsilon) R^{-2-2 n}\left(\iint_{Q_{R}}(|G|+R|F|) d z\right)^{2} .
\end{aligned}
$$

Here, we have used the fact that $\|D u(\cdot, t)\|_{L^{q}\left(B_{R}\right)}^{2} \leq C R^{n\left(\frac{2}{q}-1\right)}\|D u(\cdot, t)\|_{L^{2}\left(B_{R}\right)}^{2}$. Integrating the above over $t \in\left[-R^{2}, 0\right]$ and dividing by $R^{2}$, we prove the lemma.

Concerning weak solutions to (1.1), we also need the following result on higher integrability of their gradients. The result sharpens that of [3].

Lemma 3.5. Let $u \in V(Q)$ be a weak solution to the system

$$
u_{t}=\operatorname{div}(a(u, D u))+F(u, D u) .
$$

Assume that

$$
\begin{gathered}
a(u, D u) D u \geq \lambda|D u|^{2}, \quad|a(u, D u)| \leq \Lambda|D u|, \\
|F(u, D u)| \leq f+C|D u|,
\end{gathered}
$$

where $\lambda, \Lambda, C$ are positive constants and $f \in L^{r}(Q)$ for some $r>2$.

Then $D u \in L_{l o c}^{p}(Q)$ for some $p>2$ and there is some constant $C$ such that

$$
\left(\oiint_{Q_{R / 2}}|D u|^{p} d z\right)^{\frac{1}{p}} \leq C\left(\oiiint_{Q_{R}}|D u|^{2} d z\right)^{\frac{1}{2}}+C R\left(\oiint_{Q_{R}} f^{r} d z\right)^{\frac{1}{r}}
$$

for all $Q_{R} \subset Q$.

Proof. It is easy to see that $u$ satisfies

$$
\left|\iint_{Q_{R}} u \phi_{t} d z\right| \leq C \iint_{Q_{R}}(|G||D \phi|+|F||\phi|) d z \quad \forall \phi \in C_{0}^{1}\left(Q_{R}\right),
$$


where $G=a(u, D u)$. We then see that the result of Lemma 3.4 applies here for any $Q_{R} \subset Q$ to give

$$
\begin{aligned}
\frac{1}{R^{2}} \oiint_{Q_{R}} \mid & \left|u-u(\cdot, t)_{R}\right|^{2} d z \leq \varepsilon \int_{Q_{R}}|D u|^{2} d z \\
& +C(\varepsilon)\left[\left(\oiint_{Q_{R}}|D u|^{q} d z\right)^{\frac{2}{q}}+\left(\oiint_{Q_{R}}|G|+R|F| d z\right)^{2}\right]
\end{aligned}
$$

for any $q \in[2 n /(n+2), 2)$ and $\varepsilon>0$. Using the assumptions, we get

$$
\begin{aligned}
\frac{1}{R^{2}} \oiiint_{Q_{R}} & \left|u-u(\cdot, t)_{R}\right|^{2} d z \leq \varepsilon \int_{Q_{R}}|D u|^{2} d z \\
& +C(\varepsilon)\left[\left(\oiiint_{Q_{R}}|D u|^{q} d z\right)^{\frac{2}{q}}+\left(\oiint_{Q_{R}} \Lambda|D u| d z\right)^{2}+\oiiint_{Q_{R}} R^{2} f^{2} d z\right] .
\end{aligned}
$$

On the other hand, by testing the equation of $u$ with $(u-u(\cdot, t)) \phi^{2}$, where $\phi$ is a cutoff function for $Q_{\frac{R}{2}}$ and $Q_{R}$, we easily get (see [3]) the following Cacciopoli type inequality:

$$
\oiiint_{Q_{\frac{R}{2}}}|D u|^{2} d z \leq \frac{1}{R^{2}} \oiiint_{Q_{R}}\left|u-u(\cdot, t)_{R}\right|^{2} d z+R^{2} \oiiint_{Q_{R}}|f|^{2} d z .
$$

Combining the above, we obtain the following reverse Hölder inequality:

$$
\begin{aligned}
& \oiiint_{Q_{\frac{R}{2}}}|D u|^{2} d z \leq \varepsilon \int_{Q_{R}}|D u|^{2} d z \\
& +C(\varepsilon)\left[\left(\oiint_{Q_{R}}|D u|^{q} d z\right)^{\frac{2}{q}}+\left(\oiiint_{Q_{R}}|D u| d z\right)^{2}+\oiiint_{Q_{R}} R^{2} f^{2} d z\right] .
\end{aligned}
$$

It is now well known (see [3]) that the above gives the $L^{p}$ estimate (3.12) for some $p>2$.

Remark 3.6. The last term in (3.12) is uniformly bounded for any $R>0$ if we assume that $f$ belongs to the Morrey space $L^{r, n+2-r}(Q)$. We will later make use of the above lemma for BMO weak solutions $u$. In that case, (3.11) can be relaxed to $f \sim|u|^{q}$ for any $q>0$ and the conclusion (3.12) holds with the integral of $f$ being replaced by the BMO norm of $u$. To see this, we need only recall that $u \in L_{l o c}^{r}(Q)$ for any $r>1$ if $u$ is BMO (see Lemma 3.1). Furthermore, we can also allow $F$ to have quadratic growth in $D u$ and (3.11) to be $|F(u, D u)| \leq \varepsilon_{0}|D u|^{2}+|f|$ for some sufficiently small $\varepsilon_{0}$.

Remark 3.7. The lemma continues to hold for $u$ weakly and approximately solves (3.9) in the following sense:

$$
\left|\iint_{Q_{R}}-u \phi_{t}+\langle a(u, D u), D \phi\rangle-\langle F, \phi\rangle d z\right| \leq \delta\|D u\|_{L^{2}\left(Q_{R}\right)}\|D \phi\|_{L^{2}\left(Q_{R}\right)}
$$

for any $Q_{R} \subset Q, \phi \in C_{0}^{1}(Q)$ and $\delta$ is sufficiently small. We see that Lemma 3.4 still applies here and the Cacciopoli type inequality (3.14) then becomes

$$
\oiiint_{Q_{\frac{R}{2}}}|D u|^{2} d z \leq \delta \int_{Q_{R}}|D u|^{2} d z+\frac{1}{R^{2}} \oiiint_{Q_{R}}\left|u-u(\cdot, t)_{R}\right|^{2} d z+R^{2} \oiiint_{Q_{R}}|f|^{2} d z .
$$

The proof can continue, as $\delta$ is small, to give the $L^{p}$ estimate for $D u$. 


\section{THE APPROXIMATION}

In this section, we establish our main vehicle for the proof of our regularity results. We will show that if a vector-valued function $u$ almost and weakly solves a parabolic system, then it can be approximated by a solution $v$ to the system in a controllable way.

To begin, let us fix a cylinder $Q_{R}=B_{R} \times\left[-R^{2}, 0\right]$ and consider a collection $\mathcal{A}$ of matrix-valued functions $A(u, \zeta)$ and a collection $\mathcal{F}$ of vector-valued functions $F(u, \zeta)$ that are defined on $\mathbb{R}^{m} \times \mathbb{R}^{n m}$ and satisfy the following:

a.1) There are positive constants $\lambda, \Lambda$ such that for each $A \in \mathcal{A}$,

$$
\begin{gathered}
\langle A(u, \zeta), \zeta\rangle \geq \lambda|\zeta|^{2}, \quad|\langle A(u, \zeta), \eta\rangle| \leq \Lambda|\zeta||\eta|, \\
\left\langle\frac{\partial A}{\partial \xi}(u, \zeta) \eta, \eta\right\rangle \geq \lambda|\eta|^{2}
\end{gathered}
$$

for all $\zeta, \eta \in \mathbb{R}^{n m}, u \in \mathbb{R}^{m}$.

a.2) For each $A \in \mathcal{A}$ and any given bounded function $g \in C^{1}\left(Q_{\rho}\right)$, where $Q_{\rho} \subset Q_{R}$, the system

$\iint_{Q_{\rho}}-u \phi_{t}+\langle A(u, D u), D \phi\rangle-\langle F(u, D u), \phi\rangle d z=0, \forall \phi \in C_{0}^{1}\left(Q_{\rho}\right), \quad u=g$ on $S_{\rho}$

has a solution $u$ in $C^{1}\left(Q_{\rho}\right)$. Moreover, $\|u\|_{B M O\left(Q_{\rho}\right)} \leq C\left(\|g\|_{B M O\left(Q_{\rho}\right)}\right)$.

a.3) For any $F \in \mathcal{F}$, there are a positive constant $C$ and a function $f \in$ $L_{l o c}^{r, n+2-r}\left(\mathbb{R}^{n+1}\right)$ with $r>2$ such that

$$
\begin{gathered}
|F(u, \zeta)| \leq f+C|\zeta|, \\
|A(u, \xi)-A(v, \xi)| \leq C|u-v||\xi|, \\
|F(u, \xi)-F(v, \eta)| \leq C|u-v|+C|\xi-\eta| .
\end{gathered}
$$

We remark that a.2) is the combination of H.4) and H.5) in the previous section and it will be used later for "nice" systems, with parameters in $\mathcal{I}$. The existence result in a.2) partly comes from Amann's results and ours (see Remark 2.1). Meanwhile, only BMO norms of solutions are assumed to be uniform in a.2).

We will prove the following "nonlinear heat approximation lemma".

Proposition 4.1. Assume a.1)-a.3). For any given $M, \varepsilon>0$ and $\beta>1$ there exists $\delta \in(0,1]$ that depends only on $\lambda, \Lambda, \varepsilon, \beta$ such that if $A \in \mathcal{A}$ and $u \in V\left(Q_{R}\right) \cap$ $B M O\left(Q_{R}\right)$ satisfy

$$
\oiiint_{Q_{R}}\left|u-u_{R}\right|^{2} d z+R^{2} \oiiint_{Q_{R}}|D u|^{2} d z \leq M
$$

and

$$
\left|\iint_{Q_{R}}-u \phi_{t}+\langle A(u, D u), D \phi\rangle-\langle F(u, D u), \phi\rangle d z\right| \leq \delta\|D u\|_{L^{2}\left(Q_{R}\right)}\|D \phi\|_{L^{2}\left(Q_{R}\right)}
$$

for any $Q_{R} \subset Q, \phi \in C_{0}^{1}(Q)$, then either

$$
R^{2} \bigoplus_{Q_{R}}|D u|^{2} d z<\varepsilon
$$


or there exists $v$ in $V\left(Q_{R / 2}\right)$ such that $\|v\|_{V\left(Q_{R / 2}\right)} \leq C(\lambda, \Lambda, M)$ and

$$
\begin{aligned}
\iint_{Q_{R / 2}} & -v \phi_{t}+\langle A(v, D v), D \phi\rangle-\langle F(v, D v), \phi\rangle d z=0, \quad \forall \phi \in C_{0}^{1}\left(Q_{R / 2}\right), \\
& \left\{\begin{array}{l}
R^{-2} \oiint_{Q_{R / 2}}|v-u|^{2} d z \leq \varepsilon \oiint_{Q_{R}}|D u|^{2} d z \text { and } \\
\int_{Q_{R / 2}}|D v|^{2} d z \leq \beta \int_{Q_{R / 2}}|D u|^{2} d z .
\end{array}\right.
\end{aligned}
$$

Proof. For simplicity we will present the proof when $F$ is identically zero. It is not difficult to see that the presence of $F$, satisfying our assumptions, will introduce some extra terms, which can be treated by the same argument (see also Remark 4.2 following the proof).

The proof is by contradiction. We then assume that there exist $\varepsilon_{0}>0$ and sequences $\left\{A_{k}\right\},\left\{u_{k}\right\},\left\{\varepsilon_{k}\right\}$ and cylinders $Q_{R_{k}}\left(x_{k}, t_{k}\right)$ such that

$$
\left|\iint_{Q_{R_{k}}}-u_{k} \phi_{t}+\left\langle A_{k}\left(u_{k}, D u_{k}\right), D \phi\right\rangle d z\right| \leq \varepsilon_{k}\left\|D u_{k}\right\|_{L^{2}\left(Q_{R_{k}}\right)}\|D \phi\|_{L^{2}\left(Q_{R_{k}}\right)}
$$

for all $\phi \in V_{0}\left(Q_{R_{k}}\right)$ but

$$
\limsup _{k \rightarrow \infty} R_{k}^{2} \int_{Q_{R_{k}}}\left|D u_{k}\right|^{2} d z>0
$$

and

$$
\left\|u_{k}-v\right\|_{L^{2}\left(Q_{R_{k} / 2}\right)} \geq \varepsilon_{0} R_{k}^{2}\left\|D u_{k}\right\|_{L^{2}\left(Q_{R_{k} / 2}\right)}
$$

for all $v$ satisfying

$$
\iint_{Q_{R_{k} / 2}}-v \phi_{t}+\left\langle A_{k}(v, D v), D \phi\right\rangle d z=0 \quad \text { for all } \phi \in V_{0}\left(Q_{R_{k} / 2}\right) .
$$

We then make a change of variables:

$$
\widetilde{u}_{k}(x, t)=u_{k}\left(x_{k}+R_{k} x, t_{k}+R_{k}^{2} t\right), \quad(x, t) \in Q_{1}=B_{1}(0) \times(-1,0) .
$$

By the boundedness assumption (4.6), the norms $\left\|u_{k}\right\|_{V\left(Q_{R_{k}}\right)}$ are uniformly bounded by $M$ and so are $\left\|\widetilde{u}_{k}\right\|_{V\left(Q_{1}\right)}$. By scaling, we can assume $R=1$ in (4.11)-(4.33). This also proves that $\delta$ is independent of $R$.

For any positive real number $h$ and any function $f$ in $L^{2}\left(Q_{2 / 3}\right)$, we denote by $f_{h}=J_{h} * f$ the standard mollifier of $f$. That is, for some smooth nonnegative function $J$ with compact support in the unit ball $Q_{1}$ of $\mathbb{R}^{n+1}$ and $\|J\|_{L^{1}\left(\mathbb{R}^{n+1}\right)}=1$, we write

$$
f_{h}(Z)=J_{h} * f(Z)=\frac{1}{h^{n+1}} \iint_{Q_{2 / 3}} J\left(\frac{|Z-z|}{h}\right) f(z) d z, \quad Z \in \mathbb{R}^{n+1} .
$$

Let $\left\{h_{k}\right\}$ be some sequence of positive reals converging to 0 and $g_{k}=\left(u_{k}-\right.$ $\left.\left(u_{k}\right)_{Q_{1}}\right)_{h_{k}}$, which is in $C^{1}\left(Q_{1}\right)$. By a.2), we can find $V_{k}=U_{k}+\left(u_{k}\right)_{Q_{1}}$ that is the solution of

$$
\begin{cases}\iint_{Q_{\frac{2}{3}}}-V_{k} \phi_{t}+\left\langle A_{k}\left(V_{k}, D V_{k}\right), D \phi\right\rangle d z=0 & \forall \phi \in C_{0}^{1}\left(Q_{\frac{2}{3}}\right) \\ V_{k}=g_{k}+\left(u_{k}\right)_{1} & \text { on } S_{\frac{2}{3}} .\end{cases}
$$


The following claims provide a contradiction to (4.12) and (4.13) and prove our proposition.

Claim I: There is a constant $C$ such that $\left\|D U_{k}\right\|_{L^{2}\left(Q_{\frac{2}{3}}\right)} \leq C\left\|D u_{k}\right\|_{L^{2}\left(Q_{\frac{2}{3}}\right)}$.

Claim II: $u_{k}-U_{k} \rightarrow 0$ in $L^{2}\left(Q_{\frac{2}{3}}\right)$.

Proof of Claim I. For any $Q_{R} \subset Q_{1}, \phi$ in $C_{0}^{1}\left(Q_{R}\right)$ and any constant vector $c \in \mathbb{R}^{m}$, we have from (4.11),

$$
\mid \iint_{Q_{R}}\left[\left\langle u_{k}-c, \phi_{t}\right\rangle+\left\langle A_{k}\left(u_{k}, D u_{k}\right), D \phi\right\rangle\right] d z \leq \varepsilon_{k}\left\|D u_{k}\right\|_{L^{2}\left(Q_{R}\right)}\|D \phi\|_{L^{2}\left(Q_{R}\right)} .
$$

Let $(\alpha)_{h_{k}}=\left(A_{k}\left(u_{k}, D u_{k}\right)\right)_{h_{k}}$ and $\phi$ be in $C_{0}^{1}\left(Q_{\frac{2}{3}}\right)$. We replace $\phi$ in the above by $\phi_{-h_{k}}$, whose support is in $Q_{5 / 6}$ if $h_{k}$ is sufficiently small, to get for all $\phi \in C_{0}^{1}\left(Q_{Q_{\frac{2}{3}}}\right)$ the following:

$$
\mid \iint_{Q_{\frac{2}{3}}}\left[-\left\langle g_{k}, \phi_{t}\right\rangle+\left\langle(\alpha)_{h_{k}}, D \phi\right\rangle\right] d z \leq \varepsilon_{k}\left\|D u_{k}\right\|_{L^{2}\left(Q_{\frac{2}{3}}\right)}\|D \phi\|_{L^{2}\left(Q_{\frac{2}{3}}\right)} .
$$

For any sufficiently positive $h$ and $t \in\left(-\frac{2}{3}, 0\right)$, let $\eta(s)$ be a $C^{1}$ function such that $\eta(s)=0$ if $s<t, \eta(s)=0$ if $s>t+h$ and $\eta$ is almost linear in $(t, t+h)$. Subtracting this inequality by the equation of $U_{k}$ and testing the result with $\phi=$ $\left(U_{k}(x, s)-g_{k}(x, s)\right) \eta^{2}(s)$, which vanishes on the boundary of $Q_{\frac{2}{3}}$, we easily obtain the following when we send $h$ to 0 :

$$
\begin{gathered}
\frac{1}{2} \sup _{s \in\left[-\frac{4}{9}, t\right]} \int_{B_{\frac{2}{3}}, t}\left|U_{k}-g_{k}\right|^{2} d x+\iint_{Q_{\frac{2}{3}}^{t}}\left\langle A_{k}\left(U_{k}, D U_{k}\right), D U_{k}\right\rangle d z \\
\leq \iint_{Q_{\frac{2}{3}}^{t}}\left\langle A_{k}\left(U_{k}, D U_{k}\right), D g_{k}\right\rangle+\left\langle(\alpha)_{h_{k}}, D U_{k}-D g_{k}\right\rangle d z \\
+\varepsilon_{k}\left\|D u_{k}\right\|_{L^{2}\left(Q_{\frac{2}{3}}\right)}\|D \phi\|_{L^{2}\left(Q_{\frac{2}{3}}\right)}
\end{gathered}
$$

for any cylinder $Q_{\frac{2}{3}}^{t}=Q_{\frac{2}{3}} \cap\{(x, s): s \leq t\}$.

Since $\left|A_{k}\left(U_{k}, D U_{k}\right)\right| \leq C\left|D U_{k}\right|$, a simple use of the Young inequality and the ellipticity of $A_{k}$ give

$$
\sup _{s \in\left[-\frac{4}{9}, t\right]} \int_{B_{\frac{2}{3}}, t}\left|U_{k}-g_{k}\right|^{2} d x+\iint_{Q_{\frac{2}{3}}^{t}}\left|D U_{k}\right|^{2} d z \leq C \iint_{Q_{\frac{2}{3}}^{t}}\left[\left|D g_{k}\right|^{2}+\left|(\alpha)_{h_{k}}\right|^{2}\right] d z .
$$

Hence,

$$
\left\|D U_{k}\right\|_{L^{2}\left(Q_{\frac{2}{3}}^{t}\right)}^{2} \leq C\left[\left\|D g_{k}\right\|_{L^{2}\left(Q_{\frac{2}{3}}^{t}\right)}^{2}+\left\|(\alpha)_{h_{k}}\right\|_{L^{2}\left(Q_{\frac{2}{3}}^{t}\right)}^{2}\right] .
$$

Because $\left\|D g_{k}\right\|_{L^{2}\left(Q_{\frac{2}{3}}^{t}\right)}^{2},\left\|(\alpha)_{h_{k}}\right\|_{L^{2}\left(Q_{\frac{2}{3}}^{t}\right)}^{2} \leq C\left\|D u_{k}\right\|_{L^{2}\left(Q_{\frac{2}{3}}^{t}\right)}^{2}$, the above yields

$$
\left\|D U_{k}\right\|_{L^{2}\left(Q_{\frac{2}{3}}^{t}\right)}^{2} \leq C\left\|D u_{k}\right\|_{L^{2}\left(Q_{\frac{2}{3}}^{t}\right)}^{2} \quad \forall t \in(-1,0] .
$$

This establishes our first claim. Moreover, because $\left\|g_{k}\right\|_{V\left(Q_{\frac{2}{3}}\right)} \leq C(M)$, (4.15) also yields

$$
\iint_{Q_{\frac{2}{3}}}\left|U_{k}\right|^{2} d z \leq C(M)
$$


Proof of Claim II. Now, Lemma 3.5 and Remark 3.7 assert that $D u_{k} \in L_{l o c}^{p}$ for some $p>2$ and we have

$$
\left(\int_{Q_{\frac{2}{3}}}\left|D u_{k}\right|^{p} d z\right)^{\frac{1}{p}} \leq C\left(\oiiint_{Q_{1}}\left|D u_{k}\right|^{2} d z\right)^{\frac{1}{2}}+C(M) .
$$

For any $\rho, r \in\left(0, \frac{2}{3}\right)$, let $\phi(x, t)=\psi(x) \eta(t)$, where $\psi, \eta$ are respectively cutoff functions in $x, t$. That is, $\psi$ is a cutoff function for $B_{\frac{2}{3}-r}$ and $B_{\frac{2}{3}}$ and $\eta$ is a cutoff function for $\left[-\frac{4}{3}+\rho^{2}, 0\right]$ and $\left[-\frac{4}{3}, 0\right]$. We can assume that $|D \psi| \leq 1 / r$ and $\left|\eta_{t}\right| \leq 1 / \rho^{2}$.

We now write $H_{k}=u_{k}-V_{k}=u_{k}-\left(u_{k}\right)_{Q_{1}}-U_{k}$. Testing the equations for $V_{k}$ with $\Phi=H_{k} \phi^{2}$, and replacing $\phi$ in (4.11) by $\Phi$ and subtracting the two results, we get

$$
\left|\iint_{Q_{\frac{2}{3}}}\left[H_{k} \frac{\partial \Phi}{\partial t}+\langle\mathbf{A}, D \Phi\rangle\right] d z\right| \leq \varepsilon_{k}\left\|D u_{k}\right\|_{L^{2}\left(Q_{\frac{2}{3}}\right)}\|D \Phi\|_{L^{2}\left(Q_{\frac{2}{3}}\right)},
$$

where $\mathbf{A}=A_{k}\left(u_{k}, D u_{k}\right)-A_{k}\left(V_{k}, D V_{k}\right)=A_{k}\left(u_{k}, D u_{k}\right)-A_{k}\left(U_{k}+\left(u_{k}\right)_{Q_{1}}, D U_{k}\right)$. As before, the above then yields

$$
\begin{aligned}
\sup _{s \in\left[-\frac{4}{9}, 0\right]} \int_{B_{\frac{2}{3}}} H_{k}^{2} \phi^{2} d x+\iint_{Q_{\frac{2}{3}}}\langle\mathbf{A}, D \Phi\rangle d z \\
\leq \iint_{Q_{\frac{2}{3}}}\left|H_{k}\right|^{2} \phi\left|\frac{\partial \phi}{\partial t}\right| d z+\varepsilon_{k}\left\|D u_{k}\right\|_{L^{2}\left(Q_{\frac{2}{3}}\right)}\|D \Phi\|_{L^{2}\left(Q_{\frac{2}{3}}\right)} .
\end{aligned}
$$

We consider the second term on the left. We have

$$
\langle\mathbf{A}, D \Phi\rangle=\left\langle\mathbf{A}, D H_{k} \phi^{2}\right\rangle+\left\langle\mathbf{A}, 2 H_{k} \phi D \phi\right\rangle .
$$

We first consider the integral of the second term on the right. We now write $Q_{t}^{\prime}=Q_{\frac{2}{3}} \cap\left\{(x, s): s \leq-\frac{4}{9}+\rho^{2}\right\}$ and $Q_{x}^{\prime}=Q_{\frac{2}{3}} \cap\left\{(x, t):|x|>\frac{2}{3}-r\right\}$. Because $D \phi=0$ in $Q \backslash Q_{x}^{\prime},|D \phi| \leq 1 / r$ and $|\mathbf{A}| \leq \Lambda\left[\left|D u_{k}\right|+\left|D U_{k}\right|\right]$, we have by the Young inequality that

$$
\iint_{Q_{\frac{2}{3}}}\left\langle\mathbf{A}, H_{k} \phi D \phi\right\rangle d z \leq \iint_{Q_{x}^{\prime}} \Lambda^{2}\left[\left|D u_{k}\right|^{2}+\left|D U_{k}\right|^{2}\right] d z+C \frac{1}{r^{2}} \iint_{Q_{x}^{\prime}} H_{k}^{2} d z .
$$

Similarly, as $\phi_{t}=0$ in $Q \backslash Q_{t}^{\prime}$ and $\left|\phi_{t}\right| \leq 1 / \rho^{2}$, we also have

$$
\iint_{Q_{\frac{2}{3}}}\left|H_{k}\right|^{2} \phi\left|\frac{\partial \phi}{\partial t}\right| d z \leq C \frac{1}{\rho^{2}} \iint_{Q_{t}^{\prime}}\left|H_{k}\right|^{2} d z .
$$

We then deduce from (4.19) and the above estimates that

$$
\sup _{s \in\left[-\frac{4}{9}, 0\right]} \int_{B_{\frac{2}{3}}} H_{k}^{2} \phi^{2} d x+\iint_{Q_{\frac{2}{3}}}\left\langle\mathbf{A}, D H_{k} \phi^{2}\right\rangle d z \leq F_{k},
$$

where

$$
\begin{aligned}
F_{k}=\varepsilon_{k}\left\|D u_{k}\right\|_{L^{2}\left(Q_{\frac{2}{3}}\right)}\|D \Phi\|_{L^{2}\left(Q_{\frac{2}{3}}\right)} & +\iint_{Q_{x}^{\prime}} \Lambda^{2}\left[\left|D u_{k}\right|^{2}+\left|D U_{k}\right|^{2}\right] d z \\
& +C \frac{1}{r^{2}} \iint_{Q_{x}^{\prime}} H_{k}^{2} d z+C \frac{1}{\rho^{2}} \iint_{Q_{t}^{\prime}}\left|H_{k}\right|^{2} d z .
\end{aligned}
$$


Concerning the second term on the left of (4.20), we write

$$
\mathbf{A}=\int_{0}^{1} \frac{\partial A_{k}\left(V_{k}, s D u_{k}+(1-s) D V_{k}\right)}{\partial \xi} d s D H_{k}+A_{k}\left(u_{k}, D u_{k}\right)-A_{k}\left(V_{k}, D u_{k}\right) .
$$

By a.1), we have

$$
\begin{gathered}
\left\langle\int_{0}^{1} \frac{\partial A_{k}\left(V_{k}, s D u_{k}+(1-s) D V_{k}\right)}{\partial \xi} d s D H_{k}, D H_{k} \phi^{2}\right\rangle \geq \lambda\left|D H_{k}\right|^{2} \phi^{2}, \\
\left|\left\langle A_{k}\left(u_{k}, D u_{k}\right)-A_{k}\left(V_{k}, D u_{k}\right), D H_{k} \phi^{2}\right\rangle\right| \leq \frac{\lambda}{2}\left|D H_{k}\right|^{2} \phi^{2}+C(\lambda)\left|u_{k}-V_{k}\right|^{2}\left|D u_{k}\right|^{2} \phi^{2},
\end{gathered}
$$

where we have used (4.4) and Young's inequality. Applying Hölder's inequality to the integral of the last term, we see that

$$
\iint_{Q_{\frac{3}{4}}}\left|H_{k}\right|^{2}\left|D u_{k}\right|^{2} \phi^{2} d z \leq\left(\iint_{Q_{\frac{3}{4}}}\left(\left|H_{k}\right| \phi\right)^{2 q} d z\right)^{\frac{1}{q}}\left(\iint_{Q_{\frac{3}{4}}}\left|D u_{k}\right|^{2 p} d z\right)^{\frac{1}{p}},
$$

where $2 p$ is the exponent in the estimate for $D u_{k}$ in Lemma 3.5 and $q=p /(p-1)$. Taking any $r$ such that $2 q=2 \nu+(1-\nu) r$ for some $\nu \in(0,1)$, we recall that $u_{k}, V_{k}$ are BMO so that the $L^{r}$ norms of $u_{k}-\left(u_{k}\right)_{Q_{1}}$ and $U_{k}$ are bounded (also thanks to (4.17)). Hence, by interpolation, the first factor on the right of the above can be estimated by

$$
\left(\iint_{Q_{\frac{3}{4}}}\left(\left|H_{k}\right| \phi\right)^{r} d z\right)^{2(1-\nu)}\left(\iint_{Q_{\frac{3}{4}}}\left(\left|H_{k}\right| \phi\right)^{2} d z\right)^{2 \nu} \leq C(M, \nu)\left(\iint_{Q_{\frac{3}{4}}}\left(\left|H_{k}\right| \phi\right)^{2} d z\right)^{2 \nu} .
$$

By our assumptions (4.12) and (4.13), the integral of $\left(H_{k} \phi\right)^{2}$ is bounded from below by some positive constant $\varepsilon_{1}$. Therefore, for any given value of $\tau \in(0,1)$, the left-hand side of the above estimate is bounded from above by

$$
C\left(M, \nu, \varepsilon_{1}\right) \iint_{Q_{\frac{3}{4}}}\left(\left|H_{k}\right| \phi\right)^{2} d z .
$$

Hence, using the $L^{2 p}$ estimate for $D u_{k}$ and the Young inequality, we see that

$$
\begin{aligned}
\iint_{Q_{\frac{3}{4}}}\left|\left\langle A_{k}\left(u_{k}, D u_{k}\right)-A_{k}\left(U_{k}, D u_{k}\right), D H_{k} \phi^{2}\right\rangle\right| d z \\
\leq \iint_{Q_{\frac{3}{4}}} \frac{\lambda}{2}\left|D H_{k}\right|^{2} \phi^{2}+C\left(M, \nu, \varepsilon_{1}\right)\left|H_{k}\right|^{2} \phi^{2} d z .
\end{aligned}
$$

Note that the above argument also holds when $Q_{\frac{3}{4}}$ is replaced by $Q_{\frac{3}{4}}^{\tau}$ for any $\tau \in(0,1)$. Thus, (4.20) becomes

$$
\sup _{s \in\left[-\frac{4}{9}, \tau\right]} \int_{B_{\frac{2}{3}}} H_{k}^{2} \phi^{2} d x \leq C\left(M, \nu, \varepsilon_{1}\right) \iint_{Q_{\frac{3}{4}}^{\tau}}\left|H_{k}\right|^{2} \phi^{2} d z+F_{k} .
$$

Here, $F_{k}$ is given by (4.21). We apply the Gronwall inequality to the above to obtain

$$
\sup _{s \in\left[-\frac{4}{9}, 0\right]} \int_{B_{\frac{2}{3}}} H_{k}^{2} \phi^{2} d x \leq C\left(M, \nu, \varepsilon_{1}\right) F_{k} .
$$


We will prove that $F_{k}<\varepsilon$ for any given $\varepsilon>0$ as $k \rightarrow \infty$, thus $H_{k} \rightarrow 0$ in $L^{2}\left(Q_{\frac{2}{3}}\right)$, and complete the proof of Claim II.

Now, as

$$
\|D \Phi\|_{L^{2}\left(Q_{\frac{2}{3}}\right)}^{2} \leq C\left\|D H_{k}\right\|_{L^{2}\left(Q_{\frac{2}{3}}\right)}^{2}+C \frac{1}{r^{2}} \iint_{Q_{x}^{\prime}} H_{k}^{2} d z
$$

we have

$$
\begin{aligned}
F_{k} \leq & \varepsilon_{k} \iint_{Q_{\frac{2}{3}}}\left(\left|D u_{k}\right|^{2}+\left|D U_{k}\right|^{2}\right) d z \\
& +C \iint_{Q_{x}^{\prime}}\left[\left|D u_{k}\right|^{2}+\left|D U_{k}\right|^{2}\right] d z+C \frac{1}{r^{2}} \iint_{Q_{x}^{\prime}} H_{k}^{2} d z+C \frac{1}{\rho^{2}} \iint_{Q_{t}^{\prime}} H_{k}^{2} d z .
\end{aligned}
$$

By (4.6) and Claim I, the first term on the right tends to 0 as $k \rightarrow \infty$. We now show that, for any given $\varepsilon>0$, if $r, \rho$ are sufficiently small, then for any $k$ large,

$$
\frac{1}{r^{2}} \iint_{Q_{x}^{\prime}}\left|H_{k}\right|^{2} d z+\frac{1}{\rho^{2}} \iint_{Q_{t}^{\prime}}\left|H_{k}\right|^{2} d z<\varepsilon
$$

and

$$
\iint_{Q_{x}^{\prime}}\left(\left|D u_{k}\right|^{2}+\left|D U_{k}\right|^{2}\right) d z<\varepsilon .
$$

Concerning (4.23), as $H_{k}=\left(u_{k}-\left(u_{k}\right)_{Q_{1}}-g_{k}\right)+\left(g_{k}-U_{k}\right)$ and $u_{k}-\left(u_{k}\right)_{Q_{1}}-g_{k} \rightarrow 0$ uniformly in $L^{2}\left(Q_{\frac{2}{3}}\right)$, we need only to prove that

$$
\frac{1}{r^{2}} \iint_{Q_{x}^{\prime}}\left|g_{k}-U_{k}\right|^{2} d z+\frac{1}{\rho^{2}} \iint_{Q_{t}^{\prime}}\left|g_{k}-U_{k}\right|^{2} d z<\varepsilon
$$

uniformly in $k$ if $r, \rho$ is sufficiently small.

For the integral on $Q_{t}^{\prime}$ in (4.25), from (4.15), we find that

$$
\begin{aligned}
\frac{1}{\rho^{2}} \iint_{Q_{t}^{\prime}}\left|U_{k}-g_{k}\right|^{2} d z & \leq \sup _{s \in\left[-\frac{4}{9},-\frac{4}{9}+\rho^{2}\right]} \int_{B_{\frac{2}{3}}, s}\left|U_{k}-g_{k}\right|^{2} d x \\
& \leq C \iint_{Q_{t}^{\prime}}\left[\left|D g_{k}\right|^{2}+\left|(\alpha)_{h_{k}}\right|^{2}\right] d z .
\end{aligned}
$$

On the other hand, because $U_{k}=g_{k}$ on the lateral part of $S_{\frac{2}{3}}$, we can use the Poincaré inequality in the $x$ variable to get

$$
\frac{1}{r^{2}} \iint_{Q_{x}^{\prime}}\left|U_{k}-g_{k}\right|^{2} d z \leq \iint_{Q_{x}^{\prime}}\left|D U_{k}-D g_{k}\right|^{2} d z \leq \iint_{Q_{x}^{\prime}}\left|D g_{k}\right|^{2}+\left|D U_{k}\right|^{2} d z .
$$

By (4.15) again,

$$
\begin{aligned}
\iint_{Q_{x}^{\prime}}\left|D U_{k}\right|^{2} d z & \leq \iint_{Q_{x}^{\prime} \backslash Q_{t}^{\prime}}\left|D U_{k}\right|^{2} d z+\iint_{Q_{t}^{\prime}}\left|D U_{k}\right|^{2} d z \\
& \leq \iint_{Q_{x}^{\prime} \backslash Q_{t}^{\prime}}\left|D U_{k}\right|^{2} d z+\iint_{Q_{t}^{\prime}}\left[\left|D g_{k}\right|^{2}+\left|(\alpha)_{h_{k}}\right|^{2}\right] d z
\end{aligned}
$$

Therefore, the left-hand side of (4.25) can be estimated by

$$
C \iint_{Q_{x}^{\prime}}\left|D u_{k}\right|^{2} d z+\iint_{Q_{x}^{\prime} \backslash Q_{t}^{\prime}}\left|D U_{k}\right|^{2} d z+\iint_{Q_{t}^{\prime}}\left[\left|D g_{k}\right|^{2}+\left|(\alpha)_{h_{k}}\right|^{2}\right] d z .
$$


By (4.26), the left-hand side of (4.24) is also bounded by the same quantity. Thus, we need only prove that the above quantity can be arbitrarily small if $r, \rho$ are sufficiently small. First of all, since $D u_{k} \in L_{l o c}^{p}\left(Q_{1}\right)$ for some $p>2$ and their norms are uniformly bounded, we see that $\left|D u_{k}\right|^{2}$ converges weakly in $L_{l o c}^{1}\left(Q_{1}\right)$. By [4, Lemma 4.4, p. 126], the integral of $\left|D u_{k}\right|^{2}$ over the set $Q_{t}^{\prime}$ is small if $Q_{t}^{\prime}$ is thin or $\rho$ is small. Because $\left\|(\alpha)_{h_{k}}\right\|_{L^{2}\left(Q_{t}^{\prime}\right)}^{2}$ and $\left\|D g_{k}\right\|_{\left.L^{2}\left(Q_{t}^{\prime}\right)\right)}$ can be estimated by liminf $\left\|D u_{k}\right\|_{L^{2}\left(Q_{t}^{\prime}\right)}$ we see that the last term in (4.27) is also small. Fixing such a $\rho$, we consider the integrals of $\left|D u_{k}\right|^{2},\left|D U_{k}\right|^{2}$ in (4.27). By the same argument, the integral of $\left|D u_{k}\right|^{2}$ over the set $Q_{x}^{\prime}$ is small if $Q_{x}^{\prime}$ is thin or $r$ is small. On the other hand, since $U_{k}=g_{k}$ on the lateral boundary of $Q_{\frac{2}{3}}$, it is easy to see that the argument of the proof of Lemma 3.4 gives that

$$
\begin{aligned}
\oiiint_{Q_{\frac{R}{2}} \cap Q_{\frac{2}{3}}}\left|D U_{k}\right|^{2} d z \leq & \varepsilon \int_{Q_{R} \cap Q_{\frac{2}{3}}}\left|D U_{k}\right|^{2} d z+C\left(\int_{Q_{R} \cap Q_{\frac{2}{3}}}\left|D U_{k}\right|^{q} d z\right)^{\frac{2}{q}} \\
& +C(\varepsilon)\left(\oiint_{Q_{R} \cap Q_{\frac{2}{3}}}\left|D U_{k}\right| d z\right)^{2}+C \int_{Q_{R} \cap Q_{\frac{2}{3}}}\left|D g_{k}\right|^{2} d z
\end{aligned}
$$

for any cylinder $Q_{R}$ centered in $Q_{\frac{2}{3}}$. Because $D g_{k} \in L_{l o c}^{p}\left(Q_{1}\right)$, we see that $D U_{k} \in$ $L_{l o c}^{p}\left(Q_{\frac{2}{3}}\right)$ as well. Again we can conclude that the integral of $\left|D U_{k}\right|^{2}$ over $Q_{x}^{\prime} \backslash Q_{t}^{\prime}$ is small if $Q_{x}^{\prime}$ is thin or $r$ is small $(r<\rho)$ and $k$ is large. Therefore, the right-hand side of (4.27) can be arbitrarily small uniformly in large $k$ if $r, \rho$ are sufficiently small. As we mentioned earlier, this gives the proof of Claim II and completes our proof.

Remark 4.2. When $F$ is present, some extra terms will occur in the proof which can be treated in the same way. Nevertheless, we should note that the condition (4.3) on $F$ and Remark 3.6 guarantee that the $L^{p}\left(Q_{R}\right)$ norm of $D u_{k}$ is uniformly bounded and our proof can continue without any major changes.

The first alternative conclusion (4.8) of the above proposition is not very helpful for our main goal of getting the decay estimate D). We then consider the following assumption that $\mathcal{A}, \mathcal{F}$ are relatively sequentially compact.

a.4) For any sequences $\left\{A_{n}\right\}$ in $\mathcal{A}$ and $\left\{F_{n}\right\}$ in $\mathcal{F}$ there are subsequences $\left\{A_{n_{k}}\right\}$ and $\left\{F_{n_{k}}\right\}$, a matrix $A$ and a function $F$, and a sequence of positive reals $\left\{\varepsilon_{k}\right\}$ converging to 0 such that

$$
\left|A_{n_{k}}(u, \zeta)-A(u, \zeta)\right| \leq \varepsilon_{k}|\zeta|, \quad\left|F_{n_{k}}(u, \zeta)-F(u, \zeta)\right| \leq \varepsilon_{k}(|f|+|\zeta|)
$$

for all $u \in \mathbb{R}^{m}, \zeta \in \mathbb{R}^{n m}$. Moreover, the derivatives $\frac{\partial A_{k}(u, \zeta)}{\partial \zeta}, \frac{\partial A(u, \zeta)}{\partial \zeta}$ and $\frac{\partial F_{k}(u, \zeta)}{\partial \zeta}, \frac{\partial F(u, \zeta)}{\partial \zeta}$ exist and are continuous in $u, \zeta$.

Remark 4.3. In applications, the family $\mathcal{A}$ usually consists of matrices $A(\mu, u, \zeta)$ with $A, F$ being differentiable (or Hölder) in $\mu$. If $\frac{\partial A}{\partial \mu}$ is bounded by $\Lambda|\zeta|$, then the above assumption is trivially verified.

We have another version of Proposition 4.1. 
Proposition 4.4. Assume a.1)-a.3) and a.4). For any given $M, \varepsilon>0$ and $\beta>1$ there exists $\delta \in(0,1]$ that depends only on $\lambda, \Lambda, \varepsilon, \beta$ such that if $A \in \mathcal{A}, F \in \mathcal{F}$ and $u \in V\left(Q_{R}\right)$ satisfy

$$
\oiiint_{Q_{R}}\left|u-u_{R}\right|^{2} d z+R^{2} \oiiint_{Q_{R}}|D u|^{2} d z \leq M
$$

and

$$
\left|\iint_{Q_{R}}-u \phi_{t}+\langle A(u, D u), D \phi\rangle-\langle F(u, D u), \phi\rangle d z\right| \leq \delta\|D u\|_{L^{2}\left(Q_{R}\right)}\|D \phi\|_{L^{2}\left(Q_{R}\right)}
$$

for any $Q_{R} \subset Q, \phi \in C_{0}^{1}(Q)$, then there exists $v$ in $V\left(Q_{R / 2}\right)$ such that $\|v\|_{B M O\left(Q_{R / 2}\right)}$ $\leq C(\lambda, \Lambda, M)$ and

$$
\left\{\begin{array}{l}
R^{-2} \oiint_{Q_{R / 2}}|v-u|^{2} d z \leq \varepsilon \int_{Q_{R}}|D u|^{2} d z \text { and } \\
\int_{Q_{R / 2}}|D v|^{2} d z \leq \beta \int_{Q_{R / 2}}|D u|^{2} d z .
\end{array}\right.
$$

Moreover, $v$ satisfies

$$
\iint_{Q_{R / 2}}-v \phi_{t}+\langle\widetilde{A}(v, D v), D \phi\rangle-\langle\widetilde{F}(v, D v), \phi\rangle d z=0, \quad \forall \phi \in C_{0}^{1}\left(Q_{R / 2}\right),
$$

where either $\widetilde{A}(v, D v)=A(v, D v)$ and $\widetilde{F}(v, D v)=F(v, D v)$ or $\widetilde{A}(v, D v)=A_{0} D v$ and $\widetilde{F}(v, D v)=F_{0} D v$ for some constant matrices $A_{0}, F_{0}$.

The proof of this result partly comes from that of Proposition 4.1. We need only consider the alternative (4.8) and show that $u$ can be approximated by a solution $v$ of a system with constant coefficients. This case can be treated via the heat approximation argument in [11. In fact, a direct proof can be provided by an argument in the proof of [3, Theorem 3.1]. For the convenience of the readers, we sketch the main lines here.

Proof. For simplicity, we assume that $F \equiv 0$. The proof is by contradiction again and we will assume $R=1$ by scaling. Thanks to Proposition 4.1, if (4.31) and (4.32) (compare with (4.9) and (4.10) ) do not hold, then there are sequences $\left\{A_{k}\right\},\left\{u_{k}\right\},\left\{\varepsilon_{k}\right\}$ satisfying (4.11) and

$$
\limsup _{k \rightarrow \infty} f \int_{Q_{1}}\left|D u_{k}\right|^{2} d z=0 .
$$

By a.4) we can assume that $\left\{A_{k}\right\}$ converges to some $\bar{A}$ and (4.28) holds. Let $U_{k}=\left(u_{k}-\left(u_{k}\right)_{Q_{1}}\right) /\left\|D u_{k}\right\|_{L^{2}\left(Q_{1}\right)}$ and derive from (4.11) (due to (4.30) ) that

$$
\left|\iint_{Q_{1}}\left[\left\langle-U_{k}, \phi_{t}\right\rangle+\left\langle\hat{A}_{k}, D \phi\right\rangle\right] d z\right| \leq \varepsilon_{k}\|D \phi\|_{L^{2}\left(Q_{1}\right)}, \text { where } \hat{A}_{k}=\frac{A_{k}\left(u_{k}, D u_{k}\right)}{\left\|D u_{k}\right\|_{L^{2}\left(Q_{1}\right)}} .
$$

Because $\left\|D U_{k}\right\|_{L^{2}\left(Q_{1}\right)}=1$, by Lemma 3.3, there is $G \in V\left(Q_{1}\right)$ such that $U_{k} \rightarrow$ $G$ and $D U_{k} \rightarrow D G$ in $L^{2}\left(Q_{1}\right)$. Moreover, since $\left\|D u_{k}\right\|_{L^{2}\left(Q_{1}\right)} \rightarrow 0$ we have by Poincaré's inequality in Lemma 3.4 that $\left\|u_{k}-\left(u_{k}\right)_{Q_{1}}\right\|_{L^{2}\left(Q_{1}\right)} \rightarrow 0$. Therefore, $u_{k} \rightarrow g$ in $L^{2}\left(Q_{1}\right)$ for some constant vector $g$. 
We claim that $G$ satisfies the following system:

$$
\iint_{Q_{1}}\left[-\left\langle G, \phi_{t}\right\rangle+\langle\hat{A} D G, D \phi\rangle\right] d z=0, \quad \hat{A}=\frac{\partial \bar{A}}{\partial \zeta}(g, 0) .
$$

Letting $k \rightarrow \infty$ in (4.34), as $D \phi$ is bounded, we will show that $\hat{A}_{k} \rightarrow \hat{A} D G$ weakly in $L^{1}\left(Q_{1}\right)$. We write

$$
\hat{A}_{k}-\hat{A} D G=\frac{A_{k}\left(u_{k}, D u_{k}\right)}{\left\|D u_{k}\right\|_{L^{2}\left(Q_{1}\right)}}-\frac{\bar{A}\left(u_{k}, D u_{k}\right)}{\left\|D u_{k}\right\|_{L^{2}\left(Q_{1}\right)}}+\frac{\bar{A}\left(u_{k}, D u_{k}\right)}{\left\|D u_{k}\right\|_{L^{2}\left(Q_{1}\right)}}-\frac{\partial \bar{A}}{\partial \zeta}(g, 0) D G,
$$

and note that (4.28) gives

$$
\iint_{Q_{1}}\left|\frac{A_{k}\left(u_{k}, D u_{k}\right)}{\left\|D u_{k}\right\|_{L^{2}\left(Q_{1}\right)}}-\frac{\bar{A}\left(u_{k}, D u_{k}\right)}{\left\|D u_{k}\right\|_{L^{2}\left(Q_{1}\right)}}\right| d z \leq \varepsilon_{k} \iint_{Q_{1}} \frac{\left|D u_{k}\right|}{\left\|D u_{k}\right\|_{L^{2}\left(Q_{1}\right)}} d z \leq \varepsilon_{k} .
$$

Hence, the left-hand side integral tends to 0 as $k \rightarrow \infty$.

On the other hand,

$$
\begin{aligned}
\iint_{Q_{1}}\left\langle\frac{\bar{A}\left(u_{k}, D u_{k}\right)}{\left\|D u_{k}\right\|_{L^{2}\left(Q_{1}\right)}}-\frac{\partial \bar{A}}{\partial \zeta}(g, 0) D G, D \phi\right\rangle d z \\
\quad=\int_{0}^{1}\left[\iint_{Q_{1}}\left\langle\frac{\partial \bar{A}}{\partial \zeta}\left(u_{k}, t D u_{k}\right) \frac{D u_{k}}{\left\|D u_{2}\right\|_{L^{2}\left(Q_{1}\right)}}-\frac{\partial \bar{A}}{\partial \zeta}(g, 0) D G, D \phi\right\rangle d z\right] d t .
\end{aligned}
$$

Due to the continuity of $\frac{\partial \bar{A}}{\partial \zeta}$, we can find a concave continuous function $\omega$ satisfying $\omega(0)=0$ such that

$$
\left|\frac{\partial \bar{A}}{\partial \zeta}\left(u_{k}, t D u_{k}\right)-\frac{\partial \bar{A}}{\partial \zeta}(g, 0)\right| \leq \omega\left(\left|u_{k}-g\right|+\left|t D u_{k}-0\right|\right) .
$$

By Jensen's inequality,

$$
\iint_{Q_{1}}\left|\frac{\partial \bar{A}}{\partial \zeta}\left(u_{k}, t D u_{k}\right)-\frac{\partial \bar{A}}{\partial \zeta}(g, 0)\right|^{2} d z \leq \omega\left(\iint_{Q_{1}}\left|u_{k}-g\right|^{2} d z+t^{2} \iint_{Q_{1}}\left|D u_{k}\right|^{2} d z\right) .
$$

Therefore, $\frac{\partial \bar{A}}{\partial \zeta}\left(u_{k}, t D u_{k}\right) \rightarrow \frac{\partial \bar{A}}{\partial \zeta}(g, 0)$ strongly in $L^{2}$. Moreover, because $D U_{k} \rightarrow$ $D G$ in $L^{2}$, we see that

$$
\frac{\partial \bar{A}}{\partial \zeta}\left(u_{k}, t D u_{k}\right) \frac{D u_{k}}{\left\|D u_{2}\right\|_{L^{2}\left(Q_{1}\right)}} \rightarrow \frac{\partial \bar{A}}{\partial \zeta}(g, 0) D G, \quad \text { in } L^{1}\left(Q_{1}\right) .
$$

We conclude that the right-hand side of (4.36) converges to zero and prove (4.35).

Now, let $z_{k}$ be the solution in $Q_{\frac{1}{2}}$ to

$$
\iint_{Q_{\frac{1}{2}}}\left[-z_{k} \phi_{t}+\widetilde{A}_{k} D z_{k} D \phi\right] d z=0, \quad \widetilde{A}_{k}=\frac{\partial A_{k}}{\partial \zeta}\left(\left(u_{k}\right)_{1}, 0\right),
$$

and $z_{k}=G$ on the parabolic boundary of $Q_{\frac{1}{2}}$. Since $G$ is a weak solution to a linear system with constant coefficients in $Q_{1}, G$ is BMO bounded in $Q_{\frac{1}{2}}$ and so is $z_{k}$ (see [3]). By Cacciopoli's inequality, $\left\|D z_{k}\right\|_{L^{2}\left(Q_{\frac{1}{2}}\right)}$ is bounded. The compactness result in Lemma 3.3 then implies that $z_{k} \rightarrow Z$ and $D z_{k} \rightarrow D Z$ in $L^{2}\left(Q_{\frac{1}{2}}\right)$ for some $Z \in$ $V\left(Q_{\frac{1}{2}}\right)$. Because $\widetilde{A}_{k} \rightarrow \hat{A}$ in $L^{\infty}$, as $\left(u_{k}\right)_{Q_{1}} \rightarrow g$, we see that $Z$ satisfies (4.35) and equals $G$ on the boundary of $Q_{\frac{1}{2}}$. By uniqueness of solutions to constant coefficient 
systems, $Z=G$. Thus $\left\|U_{k}-z_{k}\right\|_{L^{2}\left(Q_{\frac{1}{2}}\right)} \rightarrow 0$. Letting $v_{k}=\left\|D u_{k}\right\|_{L^{2}\left(Q_{1}\right)} z_{k}+\left(u_{k}\right)_{Q_{1}}$ we obtain that $\left\|u_{k}-v_{k}\right\|_{L^{2}\left(Q_{\frac{1}{2}}\right)} \leq \varepsilon\left\|D u_{k}\right\|_{L^{2}\left(Q_{1}\right)}$ when $k$ is large. Furthermore, since $\left\|D z_{k}\right\|_{L^{2}\left(Q_{\frac{1}{2}}\right)}$ is bounded, we have that $\left\|D v_{k}\right\|_{L^{2}\left(Q_{\frac{1}{2}}\right)} \leq C\left\|D u_{k}\right\|_{L^{2}\left(Q_{1}\right)}$. Of course, $v_{k}$ is a solution to (4.37), a linear system with constant coefficients. Our proof is then complete.

\section{5. $I$ IS OPEN IN $[0,1]$}

We are now ready to give the proof of Theorem 2.3 which asserts that $I$ is open under the hypotheses H.1)-H.5). We consider a family of systems

$$
u_{t}=\operatorname{div}(A(\nu, u, D u))+f(\nu, u, D u) \text {, in } Q=\Omega \times(0, T) \subset \mathbb{R}^{n+1} \text { and } \nu \in I .
$$

In the proof we will need the following simple result.

Lemma 5.1. Let $\phi:(0, \infty) \rightarrow[0, \infty)$ be a function satisfying the following:

$$
\phi(\tau R) \leq K \tau^{\gamma}\left(1+\tau^{-\kappa} \varepsilon\right) \phi(R),
$$

for some positive constants $K, \gamma, \varepsilon, \kappa$ and $\tau \in\left(0, \tau_{1}\right)$.

Then for any $\alpha<\gamma$ we can find positive numbers $\tau_{0} \leq \tau_{1}$ and $\varepsilon_{0}$ depending on $K, \alpha, \gamma, \kappa$ such that if $\tau \in\left(0, \tau_{0}\right)$ and $\varepsilon \in\left(0, \varepsilon_{0}\right)$, then

$$
\phi(\tau \rho) \leq C(K, \alpha, \gamma, \kappa) \tau^{\alpha} \phi(\rho), \quad \forall \rho \leq R .
$$

Proof. To prove this for $\rho=R$, we choose $\tau_{0} \leq \tau_{1}$ and $\varepsilon_{0}$ such that $2 K \tau_{0}^{\gamma-\alpha}<1$, $\varepsilon_{0}<\tau_{0}^{\kappa}$. It is easy to see that $\phi\left(\tau_{0} R\right) \leq \tau_{0}^{\alpha} \phi(R)$. We then iterate (5.2) to have $\phi\left(\tau_{0}^{k} R\right) \leq \tau_{0}^{\alpha k} \phi(R)$ for $k=1,2, \ldots$. Finally, for any $\rho \in(0, R)$ we can find $k$ such that $\tau_{0}^{k+1} R \leq \rho<\tau_{0}^{k} R$. By taking $\tau, R$ in (5.2) to be $\rho / \tau_{0}^{k} R$ and $\tau_{0}^{k} R$, we see that $\tau_{0} \leq \tau \leq 1$ and there is a constant $K_{1}$ depending only on $K, \alpha, \gamma, \kappa$ such that $\phi(\rho) \leq K_{1} \phi\left(\tau_{0}^{k} R\right)$. Then

$$
\phi(\rho) \leq K_{1} \phi\left(\tau_{0}^{k} R\right) \leq K_{1} \tau_{0}^{\alpha k} \phi(R) \leq \frac{K_{1}}{\tau_{0}^{\alpha}}\left(\frac{\rho}{R}\right)^{\alpha} \phi(R) .
$$

This gives the claim for $\rho \leq R$ if $\tau \leq \tau_{0}$ and $\varepsilon \leq \varepsilon_{0}$.

Proof of Theorem 2.3. From the discussion following our assumptions H.1)-H.5), we need only prove that $I$ is open with respect to the property $\mathrm{D})$. In the proof below, we will make use of Proposition 4.4 and the family $\mathcal{A}, \mathcal{F}$ will be taken to be $\{A(\nu, u)\}_{\nu \in I}$ and $\{F(\nu, u, D u)\}_{\nu \in I}$. It is clear that our assumptions H.1)-H.5) imply those of Proposition 4.4

Let $\mu$ be in $I$ and $\eta$ be in $[0,1]$. We consider a bounded weak solution $u$ of (5.1) with $\nu=\eta$ and $M=\|u\|_{B M O(Q)}$. For any given $\delta>0$, by (5.1) and H.3), if $|\eta-\mu|$ is sufficiently small, then

$$
\iint_{Q_{R}}\left[-u \phi_{t}+\langle A(\mu, u, D u), D \phi\rangle-\langle F(\mu, u, D u), \phi\rangle\right] d z \leq \delta\|u\|_{V\left(Q_{2 R}\right)}\|\phi\|_{C^{1}\left(Q_{R}\right)}
$$

for all $\phi \in C_{0}^{1}\left(Q_{R}\right)$.

Thus, for any given $\varepsilon>0$ and some $\beta(M)>1$, we can apply Proposition 4.4 to find a weak solution $v$ satisfying either (5.1) with $\nu=\mu \in I$ or a system with constant coefficients such that

$$
\oiiint_{Q_{R / 2}}|u-v|^{2} d z \leq \varepsilon R^{2} \oiint_{Q_{R}}|D u|^{2} d z, \quad \oiiint_{Q_{R / 2}}|D v|^{2} d z \leq \beta(M) \oiint_{Q_{R}}|D u|^{2} d z .
$$


In both cases, $\|v\|_{B M O\left(Q_{R / 2}\right)} \leq M$ and the decay estimate (2.3) holds for $v$ because $\mu \in I$. That is, for $\alpha \in(0, \alpha(M))$ and $0<\tau<\min \{\tau(M, \alpha), 1 / 2\}, v$ satisfies

$$
\oiiint_{Q_{\tau R}}\left|v-(v)_{\tau R}\right|^{2} d z \leq C(M, \alpha) \tau^{\alpha} \oiiint_{Q_{R / 2}}\left|v-(v)_{R / 2}\right|^{2} d z .
$$

We also have

$$
\begin{aligned}
\oiiint_{Q_{\tau R}}\left|u-(u)_{\tau R}\right|^{2} d z & \leq \oiint_{Q_{\tau R}}\left|u-(v)_{\tau R}\right|^{2} d z \\
& \leq \oiiint_{Q_{\tau R}}|u-v|^{2} d z+\oiiint_{Q_{\tau R}}\left|v-(v)_{\tau R}\right|^{2} d z \\
& \leq \tau^{-n-2} \oiint_{Q_{\frac{1}{2} R}}|u-v|^{2} d z+\oiint_{Q_{\tau R}}\left|v-(v)_{\tau R}\right|^{2} d z
\end{aligned}
$$

Combining the above facts, (5.3), the Poincaré inequality for $v$ and the Cacciopoli inequality for $u$, we then have

$$
\begin{aligned}
\oiiint_{Q_{\tau R}} \mid u & -\left.(u)_{\tau R}\right|^{2} d z \leq \tau^{-n-2} \int_{Q_{\frac{1}{2} R}}|u-v|^{2} d z+\int_{Q_{\tau R}}\left|v-(v)_{\tau R}\right|^{2} d z \\
& \leq \varepsilon \tau^{-n-2} R^{2} \int_{Q_{R}}|D u|^{2} d z+C(M, \alpha) \tau^{\alpha} \oiiint_{Q_{R / 2}}\left|v-(v)_{R / 2}\right|^{2} d z \\
& \leq \varepsilon \tau^{-n-2} R^{2} \int_{Q_{R}}|D u|^{2} d z+C_{1}(M, \alpha) \tau^{\alpha} R^{2} \int_{Q_{R}}|D v|^{2} d z \\
& \leq \max \{C(M, \alpha), 1\}\left(\varepsilon \tau^{-n-2}+\tau^{\alpha}\right) R^{2} \oiiint_{Q_{R}}|D u|^{2} d z \\
& \leq K(M, \alpha) \tau^{\alpha}\left(\varepsilon \tau^{-n-2-\alpha}+1\right) \oiint_{Q_{2 R}}\left|u-(u)_{2 R}\right|^{2} d z .
\end{aligned}
$$

Let $\phi(s)=\int_{Q_{s}}\left|u-u_{s}\right|^{2} d z$. We take $\alpha$ in (5.5) to be $\left.\alpha_{0}=(\alpha(M)+\alpha) / 2\right)$ and apply Lemma 5.1 with $\gamma=\alpha_{0}, \kappa=n+2+\alpha_{0}$. We see that $\phi$ satisfies (5.2) of the lemma. Thus, if $2 K(M, \alpha) \tau_{0}^{\gamma-\alpha_{0}}=2 K(M, \alpha) \tau_{0}^{(\alpha(M)-\alpha) / 2}<1$ and $\varepsilon=\varepsilon\left(\tau_{0}\right)=\varepsilon(M, \alpha)$ such that $\varepsilon<\tau_{0}^{n+3}<\tau_{0}^{\kappa}$, then

$$
\oiiint_{Q_{\rho}}\left|u-(u)_{\tau \rho}\right|^{2} d z \leq C(M, \alpha) \tau^{\alpha} \oiiint_{Q_{\rho}}\left|u-u_{\rho}\right|^{2} d z, \quad \forall \rho \leq \tau R .
$$

Hence, (2.3) of $\mathrm{D})$ holds for $u$ again for $\tau \in(0, \tau(M, \alpha))$ if we redefine $C(M, \alpha)$, $\tau(M, \alpha)$. Therefore, $\nu \in I$ if $\mu \in I$ and $|\nu-\mu|<\theta(M, \alpha)$ for some sufficiently small $\theta(M, \alpha)$. This shows that $I$ is open with respect to the property $\mathrm{D})$. Our proof is complete.

In fact, once we know that $u$ is Hölder continuous and if $F(\nu, u, \zeta)$ is smooth in $u, \zeta$, then the exponent $\alpha$ in the property $\mathrm{D})$ can be any number in $(0,2)$. We have the following result.

Corollary 5.2. Assume further that $\frac{\partial F}{\partial \zeta}(\nu, u, \zeta), \frac{\partial F}{\partial u}(\nu, u, \zeta)$ are continuous in $u$. If $\nu \in I$ and $R$ is sufficiently small, then $u$ satisfies the decay estimate (2.3) for any $\alpha \in(0,2)$. 
Proof. We will prove that $u$ can be approximated by a weak solution to a system with constant coefficients. Because $u$ is Hölder continuous, we have

$$
\oiiint_{Q_{R}}\left|u-u_{R}\right|^{2} d z \leq \varepsilon
$$

for any given positive $\varepsilon$ if $R$ is sufficiently small.

We write

$$
\begin{gathered}
A(\nu, u, D u)=\left(\int_{0}^{1} \frac{\partial A}{\partial \zeta}(\nu, u, s D u) d s\right) D u \\
F(\nu, u, D u)=\left(\int_{0}^{1} \frac{\partial F}{\partial \zeta}(\nu, u, s D u) d s\right) D u+\left(\int_{0}^{1} \frac{\partial F}{\partial u}(\nu, s u, 0) d s\right) u,
\end{gathered}
$$

and

$$
A_{0}=\frac{\partial A}{\partial \zeta}\left(\nu,(u)_{R}, 0\right), \quad F_{0}=\frac{\partial F}{\partial \zeta}\left(\nu,(u)_{R}, 0\right), \quad F_{1}=\left(\int_{0}^{1} \frac{\partial F}{\partial u}\left(\nu, s(u)_{R}, 0\right) d s\right) .
$$

Since $\frac{\partial A}{\partial \zeta}(u, \zeta)$ is continuous in $u$ and uniformly bounded in $\zeta$, we can find a continuous and concave function $\omega_{\nu}$ with $\omega_{\nu}(0)=0$ such that

$$
\left|\frac{\partial A}{\partial \zeta}(\nu, u, s D u)-\frac{\partial A}{\partial \zeta}\left(\nu,(u)_{R}, 0\right)\right| \leq \omega_{\nu}\left(\left|u-(u)_{R}\right|^{2}\right)=\Phi .
$$

Using the $L^{p}$ estimate for $D u$ and Jensen's inequality, we have for any $\phi \in$ $C_{0}^{1}\left(Q_{R}\right)$ that

$$
\begin{aligned}
& \left|\iint_{Q_{R}}\left\langle A(\nu, u, D u)-A_{0} D u, D \phi\right\rangle d z\right| \leq\left(\iint_{Q_{R}} \Phi^{2}|D u|^{2} d z\right)^{\frac{1}{2}}\left(\iint_{Q_{R}}|D \phi|^{2} d z\right)^{\frac{1}{2}} \\
& \leq \omega_{\nu}\left(\iint_{Q_{R}}\left|u-(u)_{R}\right|^{2} d z\right)^{1-\frac{2}{p}}\left(\iint_{Q_{2 R}}|D u|^{2} d z\right)^{\frac{1}{2}}\left(\iint_{Q_{R}}|D \phi|^{2} d z\right)^{\frac{1}{2}} .
\end{aligned}
$$

Similar estimates hold for the difference between $F$ and $F_{0} D u+F_{1} u$. Hence,

$$
\left|\iint_{Q_{R}}-u \phi_{t}+\left\langle A_{0} D u, D \phi\right\rangle-\left\langle F_{0} D u+F_{1} u, \phi\right\rangle d z\right| \leq \delta\|D u\|_{L^{2}\left(Q_{2 R}\right)}\|D \phi\|_{L^{2}\left(Q_{R}\right)}
$$

for all $\phi \in C_{0}^{1}\left(Q_{1}\right)$. Here, $\delta$ can be arbitrarily small (because of (5.6) ) if we initially choose $R$ small. We then see that Proposition 4.4 applies here so that $u$ can be approximated by a weak solution $v$ to the following linear system with constant coefficients:

$$
\iint_{Q_{R}}-v \phi_{t}+\left\langle A_{0} D v, D \phi\right\rangle-\left\langle F_{0} D v+F_{1} v, \phi\right\rangle d z=0 \quad \text { for all } \phi \in C_{0}^{1}\left(Q_{R}\right) .
$$

It is well known that (see [3]) $v$ satisfies (2.3) of D) for $\alpha=2$. The proof of our Theorem 2.3 can be repeated here to see that $u$ satisfies (2.3) for any $\alpha \in(0,2)$.

Further assumptions on the smoothness of the matrix $A$ will improve the regularity of weak solutions and we will show that

Corollary 5.3. Suppose that $\frac{\partial A}{\partial \zeta}(\nu, u, \zeta), \frac{\partial F}{\partial \zeta}(\nu, u, \zeta), \frac{\partial F}{\partial u}(\nu, u, \zeta)$ are Hölder in $u$. If $\nu \in I$ and $u$ is a BMO weak solution to (5.1), then Du is Hölder continuous. 
Proof. For simplicity, we will consider only the case $F \equiv 0$. For any $R>0$ and $A_{0}$ defined as in (5.7), we consider a weak solution $v$ to

$$
\left\{\begin{array}{l}
\iint_{Q_{R}}-v \phi_{t}+\left\langle A_{0} D v, D \phi\right\rangle d z=0 \quad \text { for all } \phi \in C_{0}^{1}\left(Q_{R}\right) \\
v=u \quad \text { on } S_{R}
\end{array}\right.
$$

Let $w=u-v$. We subtract the systems for $u$ and $v$ and test the result with $w$ to get

$$
\sup _{t} \int_{B_{R}}|w|^{2} d x+\iint_{Q_{R}}\left\langle A_{0} D w, D w\right\rangle d z \leq \iint_{Q_{R}}\left|\left\langle A(\nu, u, D u)-A_{0} D u, D w\right\rangle\right| d z .
$$

Arguing as in the previous proof, we see that the left-hand side of the above can be estimated by

$$
\begin{aligned}
& \iint_{Q_{R}} \omega_{\nu}\left(\left|u-(u)_{R}\right|^{2}\right)|D u||D w| d z \\
& \quad \leq\left.\varepsilon \iint_{Q_{R}}|D w|^{2}\left|d z+C(\varepsilon) \iint_{Q_{R}} \omega_{\nu}^{2}\left(\left|u-(u)_{R}\right|^{2}\right)\right| D u\right|^{2} d z .
\end{aligned}
$$

Again, by the $L^{p}$ estimate for $D u$ and Jensen's inequality, we have

$$
\iint_{Q_{R}} \omega_{\nu}^{2}\left(\left|u-(u)_{R}\right|^{2}\right)|D u|^{2} d z \leq\left[\omega\left(\int_{Q_{R}}\left|u-u_{R}\right|^{2} d z\right)\right]^{1-\frac{2}{p}} \iint_{Q_{2 R}}|D u|^{2} d z .
$$

By our assumption, there is $\sigma>0$ such that $\omega(s) \leq C s^{\sigma}$. Making use of the decay estimate for $u$, we have

$$
\iint_{Q_{R}}|D w|^{2} d z \leq C\left(\int_{Q_{R}}\left|u-u_{R}\right|^{2} d z\right)^{\left(1-\frac{2}{p}\right) \sigma} \iint_{Q_{2 R}}|D u|^{2} d z \leq C R^{n+\alpha+\alpha\left(1-\frac{2}{p}\right) \sigma} .
$$

By Corollary 5.2, we can choose $\alpha$ near to 2 to see that

$$
\iint_{Q_{R}}|D w|^{2} d z \leq C R^{n+2+\beta}
$$

for some $\beta>0$. Now, as $v$ is a weak solution to a system with constant coefficients, it is well known that

$$
\iint_{Q_{\tau R}}\left|D v-(D v)_{\tau R}\right|^{2} d z \leq C \tau^{n+4} \iint_{Q_{R}}\left|D v-(D v)_{R}\right|^{2} d z, \quad \forall \tau \in(0,1) .
$$

From this and (5.10) we easily derive

$$
\iint_{Q_{\tau R}}\left|D u-(D u)_{\tau R}\right|^{2} d z \leq C \tau^{n+4} \iint_{Q_{R}}\left|D u-(D u)_{R}\right|^{2} d z+C R^{n+2+\beta}, \quad \forall \tau \in(0,1) .
$$

A simple iteration argument then yields

$$
\iint_{Q_{\rho}}\left|D u-(D u)_{\rho}\right|^{2} d z \leq C \rho^{n+2+\beta} \iint_{Q_{R}}\left|D u-(D u)_{R}\right|^{2} d z \quad \forall \rho \in(0, R) .
$$

This proves that $D u$ is Hölder continuous and our proof is complete. 


\section{I IS CLOSED}

In this section, we will show that the set $I$ is also closed in $[0,1]$. To proceed, we take a sequence $\left\{\nu_{k}\right\}$ in $I$ such that $\nu_{k} \rightarrow \mu$ and we will show that $\mu \in I$. Thus, let us consider a weak BMO solution $u$ to (2.2) with $\nu=\mu$. By II), there is a sequence of $C^{1}$ solutions $v_{k}$ to

$$
\begin{cases}v_{t}=\operatorname{div}(A(\nu, v, D v))+F(\nu, v, D v) & \text { in } Q_{1} \\ v=g & \text { on } S_{1}\end{cases}
$$

with $\nu=\nu_{k}$ and the data $g$ is in $C^{1}$ whose BMO norm is controlled by that of $u$. The BMO norms of $v_{k}$ are bounded uniformly in terms of that of $u$ and $D v_{k}$ converges weakly in $L^{1}\left(Q_{1}\right)$ to $D u$. We will derive uniform estimates for various integral norms of $D v$ in terms of the BMO norm of $g$. Once this is established, we obtain estimates for the derivatives of the limiting $u$ and Hölder continuity of $u$. Thus, $\mu$ is in $I$.

Fixing $\nu$ in $I$ and a solution $v$ to (6.1), we will denote $a(v, \zeta)=A(\nu, v, \zeta)$ and $F(v, \zeta)=F(\nu, v, \zeta)$. We will also omit the parameter $\nu$ in the subscripts for $\lambda_{\nu, v}, \Lambda_{\nu, v}, a_{\nu, v}$ in the sequel. As $v$ is a weak solution to a nice system, $v, D v$ are Hölder continuous and $D v$ weakly solves

$$
(D v)_{t}=\operatorname{div}\left(\frac{\partial a}{\partial \xi}(v, D v) D^{2} v+\frac{\partial a}{\partial v}(v, D v) D v\right)+\frac{\partial F}{\partial v}(v, D v) D v+\frac{\partial F}{\partial \xi}(v, D v) D^{2} v
$$

The proof of Theorem 2.5 relies mainly on the following two lemmas which establish uniform bounds for the $L^{q}$ norms of $D v$. In the proof, for simplicity, we will assume that $F(v, D v) \equiv 0$ and leave the general case to the discussion in Remark 6.3

Lemma 6.1. For any $\phi \in C_{0}^{1}\left(Q_{\frac{3}{4}}\right)$ we have

$$
\|D v \phi\|_{V(Q)} \text { and } \iint_{Q}|D v|^{\frac{2(n+2)}{n}} \phi^{2} d z \leq C\left(M_{B}\right) .
$$

Proof. Let $\phi$ be in $C_{0}^{1}\left(Q_{1}\right)$ with $\operatorname{supp} \phi \subset Q_{\frac{3}{4}}$. Testing (6.2) with $D v \phi^{2}$ and integrating by parts in $x$, we get

$$
\begin{aligned}
& \sup _{t} \int_{\Omega}|D v|^{2} \phi^{2} d x+\lambda \iint_{Q}\left|D^{2} v\right|^{2} \phi^{2} d z \\
& \quad \leq \iint_{Q} \frac{\left|a_{v}\right|^{2}}{\lambda}|D v|^{4} \phi^{2}+|D v|^{2}\left(\left|\phi_{t}\right|+\Lambda|D \phi|^{2}+1\right) d z .
\end{aligned}
$$

By Cacciopoli's inequality we note that

$$
\iint_{Q}|D v|^{2}\left(\left|\phi_{t}\right|+|D \phi|^{2}\right) d z \leq C\left(M_{B}\right)
$$

We first estimate the integral of $|D v|^{4}$ in (6.4). By integrating by parts in $x$,

$$
\iint_{Q}|D v|^{4} \phi^{2} d z=\iint_{Q} v D\left(|D v|^{3} \phi^{2}\right) d z \leq M \iint_{Q}\left(\left|D^{2} v\right||D v|^{2} \phi^{2}+|D v|^{3} \phi|D \phi|\right) d z
$$

where $M=M_{\nu, v}=\sup _{Q_{\frac{3}{4}}}|v|$.

Young's inequality applying to the right then gives

$$
\iint_{Q}|D v|^{4} \phi^{2} d z \leq \varepsilon \iint_{Q}|D v|^{4} \phi^{2} d z+C(\varepsilon) M^{2} \iint_{Q}\left(\left|D^{2} v\right|^{2} \phi^{2}+|D v|^{2}|D \phi|^{2}\right) d z .
$$


Thus, for $\varepsilon=\frac{1}{2}$, we obtain

$$
\frac{a_{v}^{2}}{\lambda} \iint_{Q}|D v|^{4} \phi^{2} d z \leq 4 \frac{a_{v}^{2} M^{2}}{\lambda} \iint_{Q}\left|D^{2} v\right|^{2} \phi^{2}+|D v|^{2}|D \phi|^{2} d z .
$$

Using this in (6.4) and the assumption on the smallness of $a_{v} M$ in (2.12), we obtain

$$
\begin{aligned}
& \sup _{t} \int_{\Omega}|D v|^{2} \phi^{2} d x+\lambda \iint_{Q}\left|D^{2} v\right|^{2} \phi^{2} d z \\
& \quad \leq \iint_{Q}|D v|^{2}\left(\left|\phi_{t}\right|+\left(\Lambda+\frac{\left|a_{v}\right|^{2} M^{2}}{\lambda}\right)|D \phi|^{2}+1\right) d z .
\end{aligned}
$$

Since

$$
\|D v \phi\|_{V(Q)}^{2} \leq \sup _{t} \int_{\Omega}|D v|^{2} \phi^{2} d x+\iint_{Q}\left(\left|D^{2} v\right|^{2} \phi^{2}+|D v|^{2}|D \phi|^{2}\right) d z,
$$

(6.6) and (6.5) give the first part of (6.3). Finally, the imbedding inequality (3.1) applied to $D v \phi$ completes the proof.

To get estimates for higher powers of $|D v|$, we need the following lemma.

Lemma 6.2. Let $\alpha$ be a positive number. Assume that $\frac{\alpha}{2+\alpha}=\delta_{\alpha, v} \frac{\lambda_{v}}{\Lambda_{v}}$ for some $\delta_{\alpha, v} \in(0,1)$ and

$$
2 a_{v} M(2+\alpha+1)<\sigma_{0} \widehat{\lambda}_{v}, \quad \text { with } \widehat{\lambda}_{v}=\left(1-\delta_{\alpha, v}^{2}\right) \lambda_{v}, \quad \sigma_{0} \in(0,1) .
$$

If $\phi \in C_{0}^{1}\left(Q_{\frac{3}{4}}\right)$, then

$$
\iint_{Q}\left(|D v|^{1+\frac{\alpha}{2}} \phi\right)^{\frac{2(n+2)}{n}} d z \leq\left(\left(1-\sigma_{0}^{2}\right) \widehat{\lambda}_{v}\right)\left(\iint_{Q}|D v|^{2+\alpha}\left(\left|\phi_{t}\right|+|D \phi|^{2}+1\right) d z\right)^{\frac{n+2}{n}} \text {. }
$$

Proof. To proceed, we recall the following facts in [7: from the ellipticity condition of $\frac{\partial a}{\partial \xi}=\left(A_{k l}^{i j}\right)$ we have for $\kappa_{v}=\lambda_{v} / \Lambda_{v}^{2}$ and $\nu_{v}=\lambda_{v} / \Lambda_{v}$ that

$$
\sum_{i, k}\left(\eta_{i}^{k}-\kappa A_{k l}^{i j} \eta_{l}^{j}\right)^{2} \leq\left(1-2 \kappa_{v} \lambda_{v}+\kappa_{v}^{2} \Lambda_{v}^{2}\right)|\eta|^{2}=\left(1-\nu_{v}^{2}\right)|\eta|^{2} .
$$

The lemma [7, p. 677] then gives $D \zeta D\left(\zeta|\zeta|^{\alpha}\right) \geq \mu^{\frac{1}{2}}(\alpha)|D \zeta|\left|D\left(\zeta|\zeta|^{\alpha}\right)\right|$ for $\zeta$ : $\mathbb{R}^{n} \rightarrow \mathbb{R}^{n m}$ and $\mu(\alpha)=1-\left(\frac{\alpha}{2+\alpha}\right)^{2}$. Therefore, with $\zeta=D v$,

$$
\begin{aligned}
\kappa_{v} \sum \frac{\partial a}{\partial \xi} D^{2} v D\left(D v|D v|^{\alpha}\right)= & {\left[\kappa_{v} \sum\left(\frac{\partial a}{\partial \xi} D^{2} v-D^{2} v\right) D\left(D v|D v|^{\alpha}\right)\right] } \\
& +D(D v) D\left(D v|D v|^{\alpha}\right) \\
\geq & \left(\mu^{\frac{1}{2}}(\alpha)-\left(1-\nu_{v}^{2}\right)^{\frac{1}{2}}\right)|D(D v)|\left|D\left(D v|D v|^{\alpha}\right)\right| .
\end{aligned}
$$

Thus, if $\frac{\alpha}{2+\alpha}=\delta_{\alpha, v} \frac{\lambda_{v}}{\Lambda_{v}}$ for some $\delta_{\alpha, v} \in(0,1)$ then the constant in front of $\left|D^{2} v\right|^{2}|D v|^{\alpha}$ is

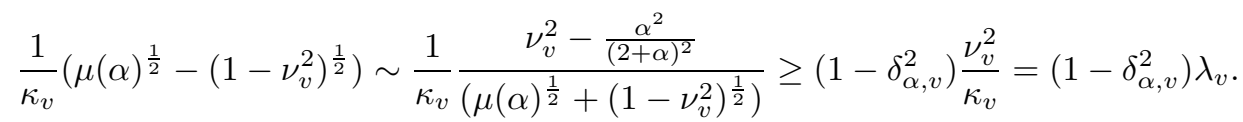

Hence,

$$
\begin{aligned}
\sum \frac{\partial a}{\partial \xi} D^{2} v D\left(D v|D v|^{\alpha}\right) & \geq \frac{1}{\kappa_{v}}\left(\mu^{\frac{1}{2}}(\alpha)-\left(1-\nu_{v}^{2}\right)^{\frac{1}{2}}\right)|D(D v)| \mid D\left(D v|D v|^{\alpha}\right) \\
& \geq \widehat{\lambda}_{v}|D v|^{\alpha}\left|D^{2} v\right|^{2} .
\end{aligned}
$$


Testing (6.2) with $D v|D v|^{\alpha} \phi^{2}$ and using the Young inequality, we obtain

$$
\begin{aligned}
\sup _{t} \int_{\Omega}|D v|^{2+\alpha} \phi^{2} d x+\widehat{\lambda}_{v} \iint_{Q}\left|D^{2} v\right|^{2}|D v|^{\alpha} \phi^{2} d z \\
\quad \leq \frac{\left|a_{v}\right|^{2}}{\widehat{\lambda}_{v}} \iint_{Q}|D v|^{4+\alpha} \phi^{2} d z+\iint_{Q}|D v|^{2+\alpha}\left(\left|\phi_{t}\right|+|D \phi|^{2}+1\right) d z .
\end{aligned}
$$

We estimate the integral of $|D v|^{4+\alpha}$. By integrating by parts in $x$,

$$
\begin{aligned}
\iint_{Q}|D v|^{4+\alpha} \phi^{2} d z & =\iint_{Q} v D\left(D v|D v|^{2+\alpha} \phi^{2}\right) d z \\
& \leq M \iint_{Q}(2+\alpha+1)\left|D^{2} v\right||D v|^{2+\alpha} \phi^{2}+|D v|^{3+\alpha} \phi|D \phi| d z .
\end{aligned}
$$

Young's inequality applying to the right then gives

$$
\begin{aligned}
\iint_{Q}|D v|^{4+\alpha} \phi^{2} d z \leq & \left(\frac{1}{2}+\varepsilon\right) \iint_{Q}|D v|^{4+\alpha} \phi^{2} d z \\
& +[2 M(2+\alpha+1)]^{2} \iint_{Q}\left|D^{2} v\right|^{2}|D v|^{\alpha} \phi^{2} d z \\
& +C(\varepsilon) M^{2} \iint_{Q}|D v|^{2+\alpha}|D \phi|^{2} d z .
\end{aligned}
$$

We choose $\varepsilon<1 / 2$ in the above. Using this in (6.9) and the assumption (6.7) on $\left|a_{v} M\right|$ and $\widehat{\lambda_{v}}$, we obtain

$$
\begin{aligned}
\sup _{t} \int_{\Omega}|D v|^{2+\alpha} \phi^{2} d x+\left(1-\sigma_{0}^{2}\right) \widehat{\lambda}_{v} & \iint_{Q}\left|D^{2} v\right|^{2}|D v|^{\alpha} \phi^{2} d z \\
& \leq C \widehat{\lambda}_{v} \iint_{Q}|D v|^{2+\alpha}\left(\left|\phi_{t}\right|+|D \phi|^{2}+1\right) d z .
\end{aligned}
$$

The above also gives a similar estimate for $\left\||D v|^{1+\frac{\alpha}{2}} \phi\right\|_{V(Q)}$. Applying the imbedding inequality (3.1) with $V=|D v|^{1+\frac{\alpha}{2}} \phi$, we get the lemma.

Proof of Theorem 2.5. By M.1) and M.2), we can choose a number $\beta>n-2$ such that

$$
2 a_{v} M(2+\beta+1)<\sigma_{0} \widehat{\lambda}_{v} \text { and } \frac{\beta}{\beta+2}=\delta^{\prime} \frac{\lambda_{v}}{\Lambda_{v}}
$$

for some $\delta^{\prime} \in(0,1)$. Clearly, we can find finitely many numbers $\alpha_{0}, \ldots, \alpha_{K}$ such that $\alpha_{k} \leq \alpha_{k+1}, \alpha_{K}=\beta$ and $2+\alpha_{k+1} \leq q_{k}=\frac{n+2}{n}\left(2+\alpha_{k}\right)$. It is easy to see that

$$
2 a_{v} M\left(2+\alpha_{k}+1\right)<\sigma_{0} \widehat{\lambda} \quad \text { and } \quad \frac{\alpha_{k}}{\alpha_{k}+2}=\delta_{s, v}^{(k)} \frac{\lambda_{v}}{\Lambda_{v}}, \quad \delta_{s, v}^{(k)} \leq \delta^{\prime}
$$

for any $k \leq K$. Starting with $\alpha=\alpha_{0}$ small $\left(2+\alpha_{0} \leq 2(n+2) / n\right)$ and using the estimate for $|D v|^{\frac{2(n+2)}{n}}$ in Lemma 6.1 we see from (6.8) that

$$
\iint_{Q_{\frac{3}{4}}}|D v|^{q_{0}} d z \leq C\left(\lambda, M_{B}\right), \quad q_{0}=\frac{n+2}{n}\left(2+\alpha_{0}\right) .
$$

The choice of $\alpha_{k}$ allows us to repeat the argument for $k=1, \ldots, K$. The functions $\phi$ in Lemma 6.2 are the cutoff functions for $Q_{R_{k}}$ and $Q_{R_{k-1}}$ with $R_{k}=\frac{3}{4}-k \frac{1}{4 K}$. 
By induction, we then have

$$
\iint_{Q_{\frac{1}{2}}}|D v|^{q_{K}} d z \leq C\left(K, \lambda, M_{B}\right), \quad q_{K}=\frac{n+2}{n}\left(2+\alpha_{K}\right)>n+2 .
$$

Now, let $u$ be a weak solution which is, by II), approximated by a sequence $\left\{v_{k}\right\}$ of weak solutions to nice systems. As $D v_{k} \rightarrow D u$ weakly in $L^{1}\left(Q_{\frac{1}{2}}\right)$, by the semicontinuity of seminorms and (6.11), we have for $2 p=q_{K}$ that

$$
\iint_{Q_{R}}|D u|^{2 p} d z \leq \liminf _{k \rightarrow \infty} \iint_{Q_{R}}\left|D v_{k}\right|^{2 p} d z \leq C\left(M_{B}\right) .
$$

Hence, for any $Q_{R} \subset Q_{\frac{1}{2}}$,

$$
\begin{aligned}
\iint_{Q_{R}}|D u|^{2} d z & \leq\left(\iint_{Q_{R}}|D u|^{2 p} d z\right)^{\frac{1}{p}}\left|Q_{R}\right|^{1-\frac{1}{p}} \\
& \leq C\left(M_{B}\right) R^{n+2-(n+2) \frac{1}{p}}=C\left(M_{B}\right) R^{n+\alpha} .
\end{aligned}
$$

Since $\alpha=2-(n+2) \frac{1}{p}$ is positive, by the Poincaré inequality in Lemma 3.4, $u$ satisfies D) and is Hölder continuous.

Remark 6.3. We now consider M.3). The presence of $f(v, D v)$ gives the following extra terms in the proof of our lemmas:

$$
\iint_{Q}|D F(v)||D v|^{1+\alpha} \phi^{2} d z \text { and } \iint_{Q}\left|D^{2} v \| D v\right|^{1+\alpha} \phi^{2} d z .
$$

The first term can be estimated by

$$
C \iint_{Q}|v|^{l}|D v|^{2+\alpha} \phi^{2} d z \leq \varepsilon \iint_{Q}\left(|D v|^{1+\frac{\alpha}{2}} \phi\right)^{q} d z+C(\varepsilon) \iint_{Q}|v|^{p l} d z,
$$

with $q=\frac{2(n+2)}{n}$ and $p=\frac{2(n+2)}{n+4}$. We then choose $\varepsilon$ small and use the fact that $v \in L^{r}(Q)$ for any large $r$. On the other hand, the second term can be treated by a simple use of Young's inequality and the proof of the lemmas can continue.

Remark 6.4. The estimates of the lemmas still hold if

$$
\left|\frac{\partial F}{\partial v}(\nu, v, \xi)\right| \leq C+C|v|^{l}+\varepsilon_{0}|\xi|^{2}, \quad\left|\frac{\partial F}{\partial \xi}(\nu, v, \xi)\right| \leq C+C|v|^{l}+\varepsilon_{0}|\xi|
$$

for some sufficiently small $\varepsilon_{0}>0$.

We conclude this section by giving the proof of Theorem 2.7 concerning (2.14) when the matrix $A$ is independent of $u$. In this case, we can obviously take $a_{v}$ to be 0 so that M.2) is not needed here.

Proof. We see that $w=v_{t}$, or more precisely the difference quotient in $t$ of $v$, weakly solves

$$
w_{t}=\operatorname{div}\left(\frac{\partial a}{\partial \xi}(D v) D w\right)+\frac{\partial F}{\partial v}(v, D v) w+\frac{\partial F}{\partial \xi}(v, D v) D w .
$$

Here, we write $a(D v)=A(D v)$. Let $\phi$ be a $C^{1}$ cutoff function as in the above proof. We note that the proof of Lemma 6.1 still applies here, without using M.1) 
and M.2), to give that $D^{2} v \phi^{2}$ is in $L^{2}(Q)$. As $w=v_{t}$ can be solved in terms of $D^{2} v$, we see that $v_{t} \phi \in L^{2}(Q)$. Testing (6.12) with $w \phi$, we again obtain

$\sup _{t} \int_{\Omega}|w|^{2} \phi^{2} d x+\lambda \iint_{Q}|D w|^{2} \phi^{2} d z \leq \iint_{Q} w^{2} \phi^{2}+|D v|^{2}\left(\left|\phi_{t}\right|+\Lambda|D \phi|^{2}+1\right) d z$.

This yields $w \phi \in L^{2(n+2) / n}(Q)$. We now choose a positive and sufficiently small $\alpha$ such that $2+\alpha \leq 2(n+2) / n$ and $\alpha /(\alpha+2)<\lambda_{v} / \Lambda_{v}$ (thus (2.11) is not used here) and test (6.12) with $w^{1+\alpha} \phi^{2}$. A similar computation as in the proof Lemma 6.2 gives

$$
\sup _{t} \int_{\Omega}|w|^{2+\alpha} \phi^{2} d x+\lambda_{v} \iint_{Q}|D w|^{2}|w|^{\alpha} \phi^{2} d z \leq C \iint_{Q}|w|^{2+\alpha}\left(\left|\phi_{t}\right|+|D \phi|^{2}+1\right) d z .
$$

Thus, for each $t,\|w(\cdot, t)\|_{L^{2+\alpha}\left(B_{\frac{1}{2}}\right)}$ is bounded. Recall that the same conclusion holds for $D v$. From our ellipticity condition on $\frac{\partial a}{\partial \zeta}$, we can write

$$
D^{2} v=\left(\frac{\partial a}{\partial \zeta}(D v)\right)^{-1}\left[F(v, D v)-v_{t}\right]
$$

We obtain that $\left\|D^{2} v(\cdot, t)\right\|_{L^{2+\alpha}\left(B_{\frac{1}{2}}\right)}$ is bounded. By Sobolev's imbedding, $\|D v(\cdot, t)\|_{L^{q}\left(B_{\frac{1}{2}}\right)}$ for some $q>4$ and $v$ is Hölder continuous in $x$ if $n \leq 4$. Note that we have not used (2.11).

If $n>4$, then the proof of Lemma 6.2 using only M.1), shows that

$$
\iint_{Q_{R}}|D v|^{2} d z \leq C R^{n+\alpha} \quad \text { for some } \alpha>0 \text { and } Q_{R} \subset Q .
$$

By the Poincaré inequality in Lemma 3.4, we see that

$$
\oiiint_{Q_{R}}\left|v-(v)_{R}\right|^{2} d z \leq C R^{\alpha} \quad \text { for some } \alpha>0 \text { and } Q_{R} \subset Q \text {. }
$$

This shows that $v$ is Hölder continuous. Our proof is complete.

\section{ACKNOWLEDGEMENT}

The author appreciates the referee's careful reading of the original manuscript and constructive comments.

\section{REFERENCES}

[1] H. Amann. Dynamic theory of quasilinear parabolic systems. III. Global existence. Math. Z., 202(1989), pp. 219-250. MR 1013086 (90i:35125)

[2] F. Duzaar and G. Mingione. Second order parabolic systems, optimal regularity, and singular sets of solutions. Ann. Inst. H. Poincaré Anal. Non Linéaire 22 (2005), no. 6, pp. 705-751. MR2172857(2008h:35139)

[3] M. Giaquinta and M. Struwe. On the partial regularity of weak solutions of nonlinear parabolic systems. Math. Z. Vol. 179 (1982), pp. 437-451. MR652852 (83f:35062)

[4] E. Giusti. Direct Methods in the Calculus of Variations. World Scientific, 2003. MR1962933 (2004g:49003)

[5] O. John and J. Stara. Some (new) counterexamples of parabolic systems. Comment. Math. Univ. Carolin., 36 (1995), pp. 503-510. MR1364491 (96j:35027)

[6] O. John and J. Stara. On the regularity of weak solutions to parabolic systems in two spatial dimensions. Comm. P.D.E., 27(1998), pp. 1159-1170. MR.1642595 (99k:35079)

[7] A. Koshelev. Regularity of solutions for some quasilinear parabolic systems. Math. Nachr., 162(1993), pp. 59-88. MR1239576(94h:35092) 
[8] E. Kalita. On the Hölder continuity of solutions of nonlinear parabolic systems. Comment. Math. Univ. Carolin., 35,4(1994), pp. 675-680. MR1321237 (96b:35097)

[9] K. H. W. Küfner. Invariant regions for quasilinear reaction-diffusion systems and applications to a two population model. NoDEA, 3(1996), pp. 421-444. MR1418589 (97m:35135)

[10] O. A. Ladyzenskaja, V. A. Solonnikov, and N. N. Ural'tseva. Linear and Quasilinear Equations of Parabolic Type. AMS Transl. Monographs, vol. 23, 1967. MR0241822 (39:3159b)

[11] D. Le. Partial regularity of solutions to a class of strongly coupled degenerate parabolic systems. Discrete Contin. Dyn. Syst. 2005, suppl., pp. 576-586. MR2192716 (2006h:35113)

[12] D. Le. Global existence for a class of strongly coupled parabolic systems. Ann. Mat. Pura Appl. (4) 185 (2006), no. 1, pp. 133-154. MR2179585(2006i:35190)

[13] D. Le. Global Existence Results for Near Triangular Nonlinear Parabolic Systems (submitted).

[14] D. Le and T. Nguyen. Everywhere regularity of solutions to a class of strongly coupled degenerate parabolic systems. Comm. Partial Differential Equations 31 (2006), no. 1-3, pp. 307-324. MR2209756 (2007b:35161)

[15] D. Le and T. Nguyen. Global existence for a class of triangular parabolic systems on domains of arbitrary dimension. Proc. Amer. Math. Soc. 133 (2005), no. 7, pp. 1985-1992. MR2137864 (2005k:35215)

[16] G. M. Lieberman. Second Order Parabolic Differential Equations. World Scientific, 1998. MR:1465184 (98k:35003)

[17] J. Necas and V. Sverak. On regularity of solutions of nonlinear parabolic systems. Ann. Scuola Norm. Sup. Pisa Cl. Sci. (4), 18 (1)(1991), pp. 1-11. MR1118218(92d:35058)

[18] N. Shigesada, K. Kawasaki and E. Teramoto. Spatial segregation of interacting species. J. Theoretical Biology, 79 (1979), pp. 83-99. MR540951 (80e:92038)

[19] J. Simon. Compact sets in the space $L^{p}(0, T ; B)$, Ann. Mat. Pura Appl. 146 (4) (1987) pp. 65-96. MR916688 (89c:46055)

[20] M. Wiegner. Global solutions to a class of strongly coupled parabolic systems. Math. Ann., 292 (1992), pp. 711-727. MR.1157322(92m:35136)

Department of Mathematics, University of Texas at San Antonio, One UtSa Circle, SAN Antonio, TEXAS 78249

E-mail address: dle@math.utsa.edu 